\title{
10
}

\section{Differential Morphology}

\section{Petros MARAgos}

National Technical University of Athens

Athens, Greece

\subsection{Introduction}

Morphological image processing has been based traditionally on modeling images as sets or as points in a complete lattice of functions and viewing morphological image transformations as set or lattice operators. Thus, so far, the two classic approaches to analyzing or designing the deterministic systems of mathematical morphology have been (1) geometry, by viewing them as image set transformations in Euclidean spaces, and (2) algebra, to analyze their properties using set or lattice theory. Geometry was used mainly for intuitive understanding, and algebra was restricted to the space domain. Despite their limitations, these approaches have produced a powerful and self-contained broad collection of nonlinear image analysis concepts, operators, and algorithms. In parallel with these directions, there is a recently growing part of morphological image processing that is based on ideas from differential calculus and dynamical systems. It combines some early ideas on using simple morpholological operations to obtain signal gradients with some recent ideas on using differential equations to model nonlinear multiscale processes or distance propagation in images. In this chapter we present a unified view of the various interrelated ideas in this area and develop some systems analysis tools in both the space and a (slope) transform domain.

The main tools of morphological image processing are a broad class of nonlinear image operators, of which the two most fundamental are dilation and erosion. The space domain in which images are defined can be either continuous, $\mathbf{E}=\mathbf{R}^{2}$ or 
discrete, $\mathbf{E}=\mathbf{Z}^{2}$. For a binary image represented by a set $S \subseteq \mathbf{E}$, its morphological dilation by another planar set $B$, denoted by $S \oplus B$, and its erosion, denoted by $S \ominus B$, are the Minkowski set operations

$$
\begin{aligned}
& S \oplus B \triangleq\{\mathbf{x}+\mathbf{b}: \mathbf{x} \in S, \mathbf{b} \in B\}, \\
& S \ominus B \triangleq\left\{\mathbf{x}: B_{+\mathbf{x}} \subseteq S\right\},
\end{aligned}
$$

where $\mathbf{x}=(x, y)$ denotes points on the plane and $B_{+\mathbf{x}} \triangleq\{\mathbf{x}+\mathbf{b}: \mathbf{b} \in B\}$ denotes set translation by a vector. The corresponding signal operations are the Minkowski dilation and erosion of an image function $f: \mathbf{E} \rightarrow \overline{\mathbf{R}}$ by another (structuring) function $g$ :

$$
\begin{aligned}
& f \oplus g(\mathbf{x}) \triangleq \bigvee_{\mathbf{y} \in \mathbf{E}} f(\mathbf{y})+g(\mathbf{x}-\mathbf{y}), \\
& f \ominus g(\mathbf{x}) \triangleq \bigwedge_{\mathbf{y} \in \mathbf{E}} f(\mathbf{y})-g(\mathbf{y}-\mathbf{x}),
\end{aligned}
$$

where $\vee$ and $\wedge$ denote supremum and infimum. The signal range is a subset of $\overline{\mathbf{R}}=\mathbf{R} \cup\{-\infty,+\infty\}$. The scalar addition in $\overline{\mathbf{R}}$ is like addition in $\mathbf{R}$ extended by the rules $r \pm \infty= \pm \infty \forall r \in \mathbf{R}$ and $(+\infty)+(-\infty)=-\infty$. In convex analysis [Roc70] and optimization, the nonlinear operation $\oplus$ is called supremal convolution, and an operation closely related to $\ominus$ is the infimal convolution

$$
f \oplus^{\prime} g(\mathbf{x}) \triangleq \bigwedge_{\mathbf{y} \in \mathbf{E}} f(\mathbf{y})+{ }^{\prime} g(\mathbf{x}-\mathbf{y})
$$

where $+^{\prime}$ is like the extended addition in $\overline{\mathbf{R}}$ except that $(+\infty)+^{\prime}(-\infty)=+\infty$. A simple case of the signal dilation and erosion results when $g$ is flat, that is, equal to 0 over its support set $B$ and $-\infty$ elsewhere. Then the weighted dilation and erosion of $f$ by $g$ reduce to the flat dilation and erosion of $f$ by $B$ :

$$
f \oplus B(\mathbf{x}) \triangleq \bigvee_{\mathbf{y} \in B} f(\mathbf{x}-\mathbf{y}), \quad f \ominus B(\mathbf{x}) \triangleq \bigwedge_{\mathbf{y} \in B} f(\mathbf{x}+\mathbf{y}) .
$$

Additionally, a wide variety of (simple or complex) parallel and/or serial interconnections of the basic morphological operations, called generally "morphological systems," have found a broad range of applications in image processing and computer vision; examples include problems in nonlinear filtering, noise suppression, contrast enhancement, geometric feature detection, skeletonization, multiscale analysis, size distributions, segmentation, and shape recognition (see [Ser82, Ser88, Mar90, Hei94, Mar98] for broad surveys and more references).

Among the very few early connections between morphology and calculus were the morphological gradients. Specifically, given a function $f: \mathbf{R}^{d} \rightarrow \mathbf{R}$, with $d=$ 1,2 , its isotropic morphological sup-derivative at a point $x$ is defined by

$$
\mathcal{M} f(x) \triangleq \lim _{r \downarrow 0} \frac{f \oplus r B(x)-f(x)}{r},
$$


where $r B=\{r b: b \in B\}$ is a $d$-dimensional disk $B$ scaled to radius $r$. The derivative $\mathcal{M}$ has been used in morphological image analysis for edge detection. It actually becomes equal to $\|\nabla f\|$ when $f$ is differentiable.

A more recent application area in which calculus-based ideas have been used in morphology is that of multiscale image analysis. Detecting features, motion, and objects as well as modeling many other information extraction tasks in image processing and computer vision has necessitated the analysis of image signals at multiple scales. Following the initial formulation of multiscale image analysis using Gaussian convolutions by Marr and his co-workers [Mar82] were two other important developments, the continuous Gaussian scale-space by Witkin [Wit83] and the observation by Koenderink [Koe84] that this scale-space can be modeled via the heat diffusion partial differential equation (PDE). Specifically, if

$$
u(x, y, t)=\iint_{\mathbf{R}^{2}} f(x-v, y-w) G_{t}(v, w) \mathrm{d} v \mathrm{~d} w
$$

is the multiscale linear convolution of an original image signal $f(x, y)$ with a Gaussian function $G_{t}(x, y)=\exp \left[-\left(x^{2}+y^{2}\right) / 4 t\right] / 4 \pi t$ whose variance $(=2 t)$ is proportional to scale $t$, then the scale-space function $u$ can be generated from the isotropic and homogeneous heat diffusion $\mathrm{PDE}^{1}$ :

$$
u_{t}=\nabla^{2} u=u_{x x}+u_{y y},
$$

with initial condition $u(x, y, 0)=f(x, y)$. The popularity of this approach is due to its linearity and its relation to the heat PDE, about which much is known from physics and mathematics. The big disadvantage of the Gaussian scale-space approach is the fact that linear smoothers blur and shift important image features, for example, edges. There is, however, a variety of nonlinear smoothing filters that can smooth while preserving important image features and can provide a multiscale image ensemble, that is, a nonlinear scale-space. A large class of such filters consists of the standard morphological openings and closings (which are serial compositions of erosions and dilations) in a multiscale formulation [Che89, Mar89, Mat75, Ser82] and their lattice extensions, of which the most notable are the reconstruction openings and closings [Sal95, Vin93]. These reconstruction filters, starting from a reference image $f$ consisting of several parts and a marker $g$ (initial seed) inside some of these parts, can reconstruct whole objects with exact preservation of their boundaries and edges. In this reconstruction process they simplify the original image by completely eliminating smaller objects inside which the marker cannot fit. A detailed discussion of reconstruction filters and their applications can be found in Chapter 9 .

Until recently the vast majority of implementations of multiscale morphological filtering had been discrete. In 1992 three teams of researchers independently published nonlinear PDEs that model the continuous multiscale morphological

\footnotetext{
${ }^{1}$ Notation for PDEs: $u_{t}=\partial u / \partial t, u_{x}=\partial u / \partial x, u_{y}=\partial u / \partial y, \nabla u=\left(u_{x}, u_{y}\right), \operatorname{div}((v, w))=$ $\nabla \cdot(v, w)=v_{x}+w_{y}$.
} 
scale-space. Specifically, Alvarez et al. [Alv92, Alv93] obtained PDEs for multiscale flat dilation and erosion by compact convex structuring sets as part of their general work on developing PDE-based models for multiscale image processing that satisfy certain axiomatic principles. Brockett and Maragos [Bro92, Bro94] developed nonlinear PDEs that model multiscale morphological dilation, erosion, opening, and closing by compact support structuring elements that are either convex sets or concave functions and may have nonsmooth boundaries. Their work was based on the semigroup structure of the multiscale dilation and erosion operators and the use of morphological derivatives to deal with the development of shocks. Boomgaard and Smeulders [Boo92, Boo94] obtained PDEs for multiscale dilation and erosion by studying the propagation of the boundaries of 2D sets and signal graphs under multiscale dilation and erosion. Their work applies to convex structuring elements whose boundaries contain no linear segments, are smooth, and possess a unique normal at each point.

To illustrate the basic idea behind morphological PDEs, we consider a 1D example, for which we define the multiscale flat dilation and erosion of a 1D signal $f(x)$ by the set $[-t, t]$ as the scale-space functions

$$
\delta(x, t)=\bigvee_{|y| \leq t} f(x-y), \quad \varepsilon(x, t)=\bigwedge_{|y| \leq t} f(x+y) .
$$

An example is shown in Fig. 10.1. The PDEs generating these multiscale flat dilations and erosions are

$$
\begin{aligned}
\delta_{t} & =\left|\delta_{x}\right|, \quad \varepsilon_{t}=-\left|\varepsilon_{x}\right|, \\
\delta(x, 0) & =\varepsilon(x, 0)=f(x) .
\end{aligned}
$$

In parallel to the development of the above ideas, there have been some advances in the field of differential geometry for evolving curves or surfaces using level set methods. Specifically, Osher and Sethian [Osh88, Set96] have developed PDEs of the Hamilton-Jacobi type to model the propagation of curves embedded as level curves (isoheight contours) of functions evolving in scale-space. Further, to solve these PDEs they developed robust numerical algorithms based on stable and shock-capturing schemes that had been formulated to solve similar shockproducing nonlinear wave PDEs [Lax73]. Kimia et al. [Kim90, Tek98] have applied and extended these curve evolution ideas to shape analysis in computer vision. Arehart et al. [Are93] and Sapiro et al. [Sap93] implemented continuous-scale morphological dilations and erosions using the numerical algorithms of curve evolution to solve the PDEs for multiscale dilation and erosion.

Multiscale dilations and erosions of binary images can also be obtained via distance transforms, that is, the distance function from the original image set. Discrete distance transforms can be implemented quickly via 2D min-sum difference equations, which are special cases of recursive erosions, developed by Rosenfeld and Pfaltz [Ros66] and Borgefors [Bor86]. Using Huygen's construction, the boundaries of multiscale dilations-erosions by disks can also be viewed as the wavefronts of a wave initiating from the original image boundary and propagating 


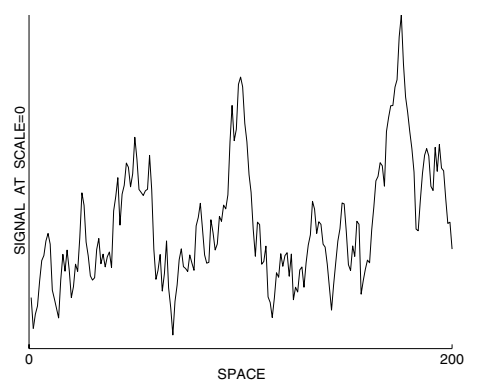

(a)

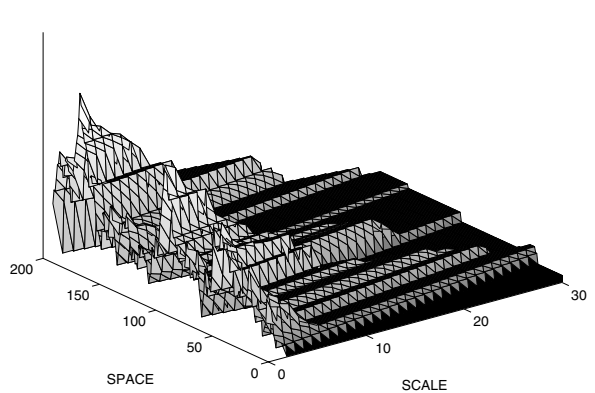

(b)

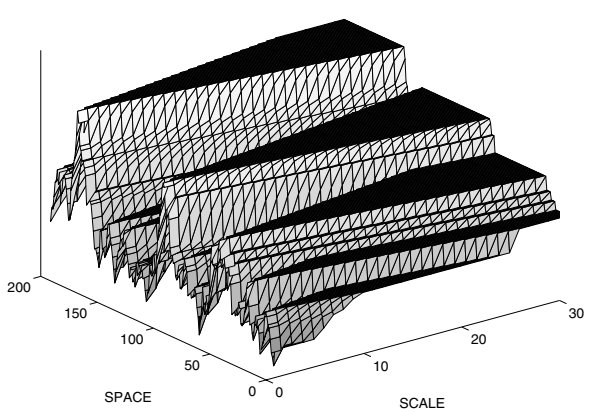

(c)

Figure 10.1: (a) Original 1D signal $f(x)$ at scale $t=0$, (b) multiscale erosion $\varepsilon(x, t)=$ $f \ominus t B(x)$, and (c) multiscale dilation $\delta(x, t)=f \oplus t B(x)$ of $f(x)$ by a set $B=[-1,1]$ for scales $t=[0,30]$.

with constant normal speed in a homogeneous medium [Blu73]. This idea can also be extended to heterogeneous media by using a weighted distance function, in which the weights are inversely proportional to the propagation speeds [Lev70]. In geometrical optics, the distance wavefronts are obtained from the isolevel contours of the solution of the eikonal PDE. This ubiquitous PDE has been applied to solving various problems in image analysis and computer vision such as shapefrom-shading, gridless halftoning, and image segmentation.

In this chapter we discuss some close relationships between the morphological derivatives, the PDEs for multiscale morphology, the eikonal PDE of optics, and the difference equations used to implement distance transforms. The unifying theme is a collection of nonlinear differential-difference equations modeling the scale or space dynamics of morphological systems. We call this area differential morphology. Whereas classical morphological image processing is based on set and lattice theory, differential morphology offers calculus-based tools and some exciting connections to the physics of wave propagation. Some additional material on nonlinear PDEs and curve evolution as applied to image processing can be found in Chapter 8.

We also present analysis tools for the nonlinear systems used in differential morphology, which have many similarities with the tools used to analyze linear 
differential schemes. These tools apply either to the space domain or to a new transform domain, the slope domain. To understand their behavior in the slope domain, we discuss some nonlinear signal transforms, called slope transforms, whose properties and applications to morphological systems have some interesting conceptual similarities with Fourier transforms and their application to linear systems.

The chapter is organized as follows. We begin in Sec. 10.2 by presenting analytic methods for 2D morphological systems both in the spatial domain, using their impulse response and generating 2D nonlinear difference equations, as well as in the slope domain, using slope transforms. In Sec. 10.3 we discuss the basic PDEs for multiscale dilations and erosions, refine them using morphological derivatives, give their slope domain interpretation, describe PDEs for opening filters, and outline numerical algorithms for their implementation. Section 10.4 summarizes the main ideas from curve evolution as they apply to differential morphology. Section 10.5 deals with distance transforms for binary images and the analysis of their computation methods based on min-sum difference equations and slope filters. Finally, the eikonal PDE, its solution via weighted distance transforms, and some of its applications to image processing are discussed in Sec. 10.6.

\subsection{D Morphological Systems and Slope Transforms}

\subsubsection{D Morphological Systems}

A 2D signal operator or system $\Psi$ is generally called

dilation if $\Psi\left(\bigvee_{i} f_{i}\right)=\bigvee_{i} \Psi\left(f_{i}\right)$ for any signal collection $\left\{f_{i}\right\}$;

erosion if $\Psi\left(\bigwedge_{i} f_{i}\right)=\bigwedge_{i} \Psi\left(f_{i}\right)$;

shift-invariant if $\Psi[f(\mathbf{x}-\mathbf{y})]=\Psi(f)(\mathbf{x}-\mathbf{y})$ for any signal $f(\mathbf{x})$ and shift $\mathbf{y}$;

translation-invariant if $\Psi[c+f(\mathbf{x}-\mathbf{y})]=c+\Psi(f)(\mathbf{x}-\mathbf{y})$, for any $f$, $\mathbf{y}$ and any constant $c$.

Of particular interest in this chapter are operators $\mathcal{E}$ that are erosion and translationinvariant (ETI) systems. Such systems are shift-invariant and obey an infimum-ofsums superposition:

$$
\mathcal{E}\left[\bigwedge_{i} c_{i}+f_{i}(\mathbf{x})\right]=\bigwedge_{i} c_{i}+\mathcal{E}\left[f_{i}(\mathbf{x})\right] .
$$

Similarly, dilation and translation-invariant (DTI) systems are shift-invariant and obey a supremum-of-sums superposition as in Eq. (10.10) but with $\bigwedge$ replaced by $\checkmark$. Two elementary signals useful for analyzing such systems are the zero impulse $\xi(\mathbf{x})$ and the zero step $\zeta(\mathbf{x})$ :

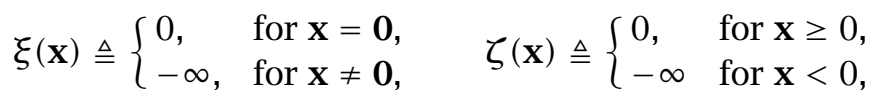


where $\mathbf{x}=(x, y) \geq 0$ means that both $x$, and $y$ are greater than or equal to 0 . Occasionally we shall refer to $\xi$ as an "upper" impulse and to its negated version $-\xi$ as a "lower" impulse. A signal can be represented as a sup or inf of weighted upper or lower impulses; for example,

$$
f(\mathbf{x})=\bigwedge_{\mathbf{y}} f(\mathbf{y})+{ }^{\prime}[-\xi(\mathbf{x}-\mathbf{y})] .
$$

If we define the lower impulse response of an ETI system $\mathcal{E}$ as its output $g_{\wedge}=$ $\mathcal{E}(-\xi)$ when the input is the zero lower impulse, we find that the system's action is equivalent to the infimal convolution of the input with its lower impulse response [Mar94]:

$$
\mathcal{E} \text { is ETI } \Longleftrightarrow \mathcal{E}(f)=f \oplus^{\prime} g_{\wedge}, \quad g_{\wedge} \triangleq \mathcal{E}(-\xi) .
$$

Similarly, a system $\mathcal{D}$ is DTI iff $\mathcal{D}(f)=f \oplus \mathcal{g}_{\vee}$, where $g_{\vee} \triangleq \mathcal{D}(\xi)$ is the system's upper impulse response. Thus, DTI and ETI systems are uniquely determined in the spatial domain by their impulse responses, which also control their causality and stability [Mar94].

To create a transform domain for morphological systems, we first note that the planes $f(\mathbf{x})=\mathbf{s} \cdot \mathbf{x}+c$ are eigenfunctions of any DTI system $\mathcal{D}$ or ETI system $\mathcal{E}$ since

$$
\begin{aligned}
& \mathcal{D}[\mathbf{s} \cdot \mathbf{x}+c]=\mathbf{s} \cdot \mathbf{x}+c+G_{\vee}(\mathbf{s}), \\
& \mathcal{E}[\mathbf{s} \cdot \mathbf{x}+c]=\mathbf{s} \cdot \mathbf{x}+c+G_{\wedge}(\mathbf{s})
\end{aligned}
$$

where $\mathbf{s} \cdot \mathbf{x} \triangleq s_{1} x+s_{2} y$ for $\mathbf{s}=\left(s_{1}, s_{2}\right)$ and $\mathbf{x}=(x, y)$ in $\mathbf{R}^{2}$ and where

$$
G_{\vee}(\mathbf{s}) \triangleq \bigvee_{\mathbf{x}} g_{\vee}(\mathbf{x})-\mathbf{s} \cdot \mathbf{x}, \quad G_{\wedge}(\mathbf{s}) \triangleq \bigwedge_{\mathbf{x}} g_{\wedge}(\mathbf{x})-\mathbf{s} \cdot \mathbf{x}
$$

are the corresponding eigenvalues, called, respectively, the upper and lower slope responses of the DTI and ETI systems. They measure the amount of shift in the intercept of the input hyperplanes with slope vector $\mathbf{s}$. They are also conceptually similar to the frequency response of linear systems.

\subsubsection{Slope Transforms}

Viewing the 2D slope response as a signal transform with variable slope vector, we define for any 2D signal $f(\mathbf{x})$ its upper slope transform as the 2D function $F_{\vee}: \mathbf{R}^{2} \rightarrow \overline{\mathbf{R}}$, defined by

$$
F_{\vee}(\mathbf{s}) \triangleq \bigvee_{\mathbf{x} \in \mathbf{R}^{2}} f(\mathbf{x})-\mathbf{s} \cdot \mathbf{x}
$$

and as its lower slope transform ${ }^{2}$ the function

$$
F_{\wedge}(\mathbf{s}) \triangleq \bigwedge_{\mathbf{x} \in \mathbf{R}^{2}} f(\mathbf{x})-\mathbf{s} \cdot \mathbf{x} .
$$

\footnotetext{
${ }^{2}$ In convex analysis [Roc70], given a convex function $h$ there uniquely corresponds another convex function $h^{*}(\mathbf{s})=\bigvee_{\mathbf{x}} \mathbf{s} \cdot \mathbf{x}-h(\mathbf{x})$, called the Fenchel conjugate of $h$. The lower slope transform of $h$ and its conjugate function are closely related since $h^{*}(\mathbf{s})=-H_{\wedge}(\mathbf{s})$.
} 


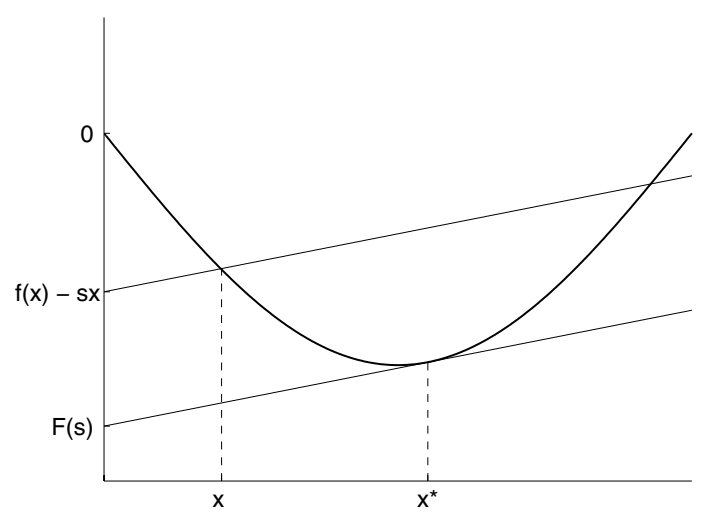

Figure 10.2: Convex signal $f$, its tangent with slope $s$, and a line parallel to the tangent.

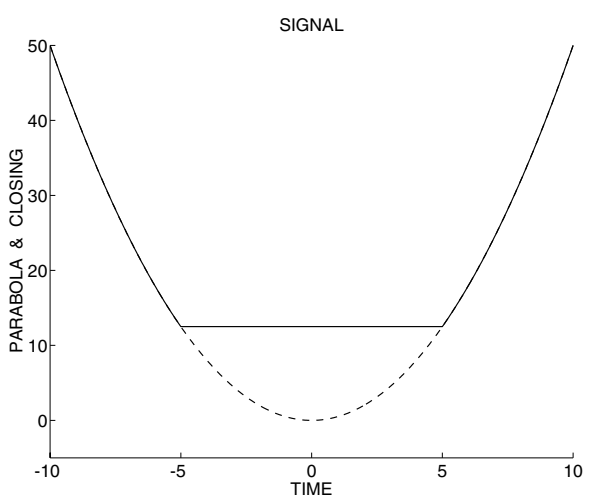

(a)

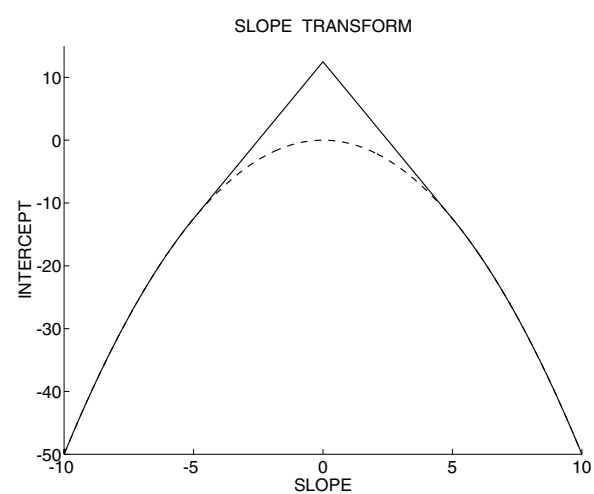

(b)

Figure 10.3: (a) Convex parabola signal $f(x)=x^{2} / 2$ (dashed line) and its morphological closing (solid line) by a flat structuring element $[-5,5]$. (b) Lower slope transform $F_{\wedge}(s)=$ $-s^{2} / 2$ of the parabola (dashed line) and of its closing (solid line).

As shown in Fig. 10.2 for a 1D signal $f(x), f(x)-s x$ is the intercept of a line with slope $s$ passing from the point $(x, f(x))$ on the signal's graph. Hence, for each $s$, the lower slope transform of $f$ is the minimum value of this intercept. For differentiable ${ }^{3}$ D signals, this minimum occurs when the above line becomes a tangent; for 2D signals the tangent line becomes a tangent plane. Examples of lower slope transforms are shown in Fig. 10.3.

\footnotetext{
${ }^{3}$ For differentiable signals, the maximization or minimization of the intercept $f(\mathbf{x})-\mathbf{s} \cdot \mathbf{x}$ involved in both slope transforms can also be done, for a fixed $\mathbf{s}$, by finding its value at the stationary point $\mathbf{x}^{*}$ such that $\nabla f\left(\mathbf{x}^{*}\right)=\mathbf{s}$. This extreme value of the intercept (as a function of the slope $\mathbf{s}$ ) is the Legendre transform [Cou62] of the signal $f$. If $f$ is convex (or concave) and has an invertible gradient, its Legendre transform is single-valued and equal to the lower (or upper) transform; otherwise, the Legendre transform is multivalued. This possibly multivalued transform has been defined as a "slope transform" [Dor94] and its properties are similar to those of the upper-lower slope transforms, but there are also some important differences [Mar94].
} 


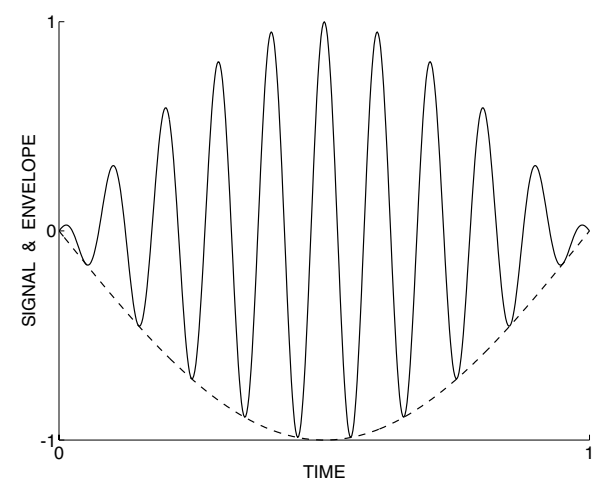

(a)

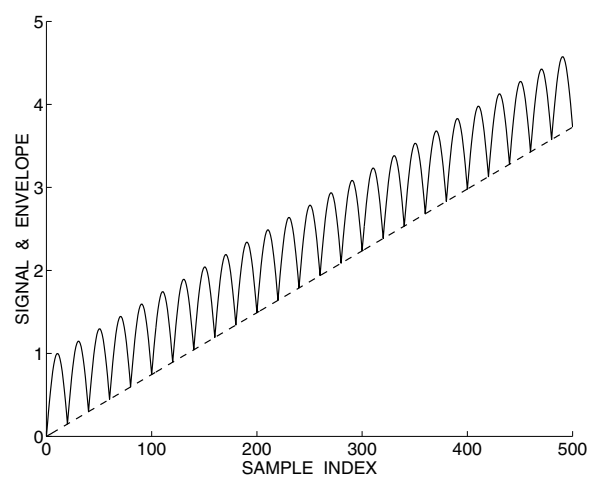

(b)

Figure 10.4: Signals $f$ (solid lines) and their lower envelopes $\breve{f}$ (dashed lines) obtained via the composition of the lower slope transform and its inverse. (a) Cosine whose amplitude has been modulated by a slower cosine pulse. (b) Impulse response $f$ of a discrete ETI system generated by the min-sum difference equation $f[n]=\min \left(-\xi[n], \wedge_{1 \leq k \leq 20} f[n-\right.$ $\left.k]+a_{k}\right)$, where $a_{k}=\sin (\pi k / 21)$.

In general, a 2D signal $f(\mathbf{x})$ is covered from above by all planes $F_{\mathrm{V}}(\mathbf{s})+\mathbf{s} \cdot \mathbf{x}$ whose infimum creates an upper envelope,

$$
\hat{f}(x) \triangleq \bigwedge_{\mathbf{s} \in \mathbf{R}^{2}} F_{\vee}(\mathbf{s})+\mathbf{s} \cdot \mathbf{x},
$$

and from below by planes $F_{\wedge}(\mathbf{s})+\mathbf{s} \cdot \mathbf{X}$ whose supremum creates the lower envelope

$$
\check{f}(x) \triangleq \bigvee_{\mathbf{s} \in \mathbf{R}^{2}} F_{\wedge}(\mathbf{s})+\mathbf{s} \cdot \mathbf{x} .
$$

We view the signal envelopes $\hat{f}(x)$ and $\breve{f}(x)$ as the "inverse" upper and lower slope transforms of $f(\mathbf{x})$, respectively. Examples are shown in Fig. 10.4. The upper (lower) slope transform is always a convex (concave) function. Similarly, the upper (lower) envelope created by the "inverse" upper (lower) slope transform is always a concave (convex) function. Further, for any signal $f$,

$$
\check{f} \leq f \leq \hat{f} \text {. }
$$

Tables 10.1 and 10.2 list several properties and examples of the 2D upper slope transform. The most striking is that supremal convolution in the time-space domain corresponds to addition in the slope domain. Note the analogy with linear systems, in which linearly convolving two signals in space corresponds to multiplying their Fourier transforms. Very similar properties also hold for the 2D lower slope transform, the only differences being the interchange of suprema with infima, concave with convex, and the supremal $\oplus$ with the infimal convolution $\oplus^{\prime}$. by

Given a discrete-domain 2D signal $f(i, j)$, we define its lower slope transform

$$
F_{\wedge}\left(s_{1}, s_{2}\right)=\bigwedge_{i=-\infty}^{\infty} \bigwedge_{j=-\infty}^{\infty} f(i, j)-\left(i s_{1}+j s_{2}\right)
$$


Table 10.1: Properties of 2D Upper Slope Transform

\begin{tabular}{|c|c|}
\hline Signal $f(\mathbf{x})^{a}$ & Transform $F_{\vee}(\mathbf{s})^{b}$ \\
\hline$\bigvee_{i} c_{i}+f_{i}(\mathbf{x})$ & $V_{i} c_{i}+F_{i}(\mathbf{s})$ \\
\hline$f\left(\mathbf{x}-\mathbf{x}_{0}\right)$ & $F(\mathbf{s})-\mathbf{s} \cdot \mathbf{x}_{0}$ \\
\hline$f(\mathbf{x})+\mathbf{s}_{0} \cdot \mathbf{x}$ & $F\left(\mathbf{s}-\mathbf{s}_{0}\right)$ \\
\hline$f(r \mathbf{x}), \quad r \in \mathbf{R}$ & $F(\mathbf{s} / r)$ \\
\hline$r f(\mathbf{x}), \quad r>0$ & $(F+G)(\mathbf{s})$ \\
\hline$(f \oplus g)(\mathbf{x})$ & $F(\mathbf{s}) \leq G(\mathbf{s}) \quad \forall \mathbf{s}$ \\
\hline$f(\mathbf{x}) \leq g(\mathbf{x}) \quad \forall \mathbf{x}$ & $G(\mathbf{s})=F(\mathbf{s}) \oplus^{\prime} r\|\mathbf{s}\|_{q}, \quad \frac{1}{p}+\frac{1}{q}=1$ \\
\hline$g(\mathbf{x})=\left\{\begin{array}{l}f(\mathbf{x}), \quad\|\mathbf{x}\|_{p} \leq r \\
-\infty, \quad\|\mathbf{x}\|_{p}>r\end{array}\right.$ & ${ }^{b} \mathbf{s}=\left(s_{1}, s_{2}\right) \in \mathbf{R}^{2}$ \\
\hline \hline${ }^{a} \mathbf{x}=(x, y) \in \mathbf{R}^{2}$ &
\end{tabular}

Table 10.2: Examples of 2D Upper Slope Transforms

\begin{tabular}{|c|c|}
\hline Signal $f(\mathbf{x})$ & Transform $F_{\vee}(\mathbf{s})$ \\
\hline $\mathbf{s}_{0} \cdot \mathbf{x}$ & $-\xi\left(\mathbf{s}-\mathbf{s}_{0}\right)$ \\
\hline $\mathbf{s}_{0} \cdot \mathbf{x}+\zeta(\mathbf{x})$ & $-\zeta\left(\mathbf{s}-\mathbf{s}_{0}\right)$ \\
\hline$\xi\left(\mathbf{x}-\mathbf{x}_{0}\right)$ & $-\mathbf{s} \cdot \mathbf{x}_{0}$ \\
\hline$\zeta\left(\mathbf{x}-\mathbf{x}_{0}\right)$ & $-\mathbf{s} \cdot \mathbf{x}_{0}-\zeta(\mathbf{s})$ \\
\hline$\left\{\begin{array}{l}\|\mathbf{x}\|_{p} \leq r, \quad p \geq 1 \\
-\infty, \quad\|\mathbf{x}\|_{p}>r, \quad p\end{array}\right.$ & $\left\{\begin{array}{l}0, \quad\|\|_{q}, \quad \frac{1}{p}+\frac{1}{q}=1 \\
+\infty, \quad\|\mathbf{s}\|_{q} \leq s_{0}\end{array}\right.$ \\
\hline$-s_{0}\|\mathbf{x}\|_{p}, \quad s_{0}>0$ & $\sqrt{1+s_{1}^{2}+s_{2}^{2}}$ \\
\hline$\sqrt{1-x^{2}-y^{2}}, \quad x^{2}+y^{2} \leq 1$ & $\left(s_{1}^{2}+s_{2}^{2}\right) / 2$ \\
\hline$-\left(x^{2}+y^{2}\right) / 2$ & $\left(\left|s_{1}\right|^{q}+\left|s_{2}\right|^{q}\right) / q$ \\
\hline$-\left(|x|^{p}+|y|^{p}\right) / p, \quad p>1$
\end{tabular}

and likewise for its upper slope transform using $\bigvee$. The properties of these slope transforms for signals defined on the discrete plane are almost identical to the ones for signals defined on $\mathbf{R}^{2}$ (see [Mar94] for details).

A more general treatment of upper and lower slope transforms on complete lattices can be found in Heijmans and Maragos [Hei97].

\subsubsection{Min-Sum Difference Equations and Discrete Slope Filters}

The space dynamics of a large class of 2D discrete ETI systems can be described by the following general 2D min-sum difference equation:

$$
u(i, j)=\left(\bigwedge_{(k, \ell) \in M_{\mathrm{o}}} a_{k \ell}+u(i-k, j-\ell)\right) \wedge\left(\bigwedge_{(k, \ell) \in M_{\mathrm{i}}} b_{k \ell}+f(i-k, j-\ell)\right),
$$


which we view as a 2D discrete nonlinear system, mapping the input signal $f$ to the output $u$. The masks $M_{\mathrm{o}}, M_{\mathrm{i}}$ are pixel coordinate sets that determine which output and input samples will be added with constant weights to form the current output sample. Similarly, the dynamics of DTI systems can be described by max-sum difference equations as in Eq. (10.18) but with $\wedge$ replaced by $\vee$.

As explained by Dudgeon and Mersereau [Dud84], for 2D linear difference equations the recursive computability of Eq. (10.18) depends on (1) the shape of the output mask $M_{\mathrm{o}}=\left\{(k, \ell): a_{k \ell}<+\infty\right\}$ determining which past output samples are involved in the recursion, (2) the boundary conditions, that is, the locations and values of the output samples $u(i, j)$ that are prespecified as initial conditions, and (3) the scanning order in which the output samples should be computed. We assume boundary conditions of value $+\infty$ and of a shape (dependent on $M_{\mathrm{o}}$ and the scanning order) appropriate so that the difference equation is an ETI system recursively computable. Obviously, $(0,0) \notin M_{0}$. The nonrecursive part of Eq. (10.18) represents an infimal convolution of the input array $f(i, j)$ with the $2 \mathrm{D}$ finitesupport structuring function $b(i, j)=b_{i j}$, which is well understood. Thus, we henceforth focus only on the recursive version of Eq. (10.18) by setting $b_{k \ell}=+\infty$, except for $b_{00}=0$. This yields the autoregressive 2D min-sum difference equation,

$$
u(i, j)=\left(\bigwedge_{(k, \ell) \in M_{0}} a_{k \ell}+u(i-k, j-\ell)\right) \wedge f(i, j) .
$$

If $g=\mathcal{E}(-\xi)$ is the impulse response of the corresponding ETI system $\mathcal{E}: f \mapsto u$, then $u=f \oplus^{\prime} g$. Finding a closed-formula expression for $g$ is generally not possible. However, we can first find the slope response $G$ and then, via the inverse lower slope transform, find the impulse response $g$ or its envelope $\breve{g}$. (For notational simplicity, we have dropped here the subscript $\wedge$ from $g$ and $G$.) Let us consider the 2D finite-support signal of the mask coefficients,

$$
a(i, j) \triangleq \begin{cases}a_{i j} & \text { for }(i, j) \in M_{\mathrm{o}} \\ +\infty & \text { otherwise }\end{cases}
$$

and its lower slope transform,

$$
A_{\wedge}\left(s_{1}, s_{2}\right)=\bigwedge_{(i, j) \in M_{0}} a_{i j}-i s_{1}-j s_{2} .
$$

Then, rewriting Eq. (10.19) as

$$
u=\left(u \oplus^{\prime} a\right) \wedge f
$$

and applying lower slope transforms to both sides yields

$$
U_{\wedge}(\mathbf{s})=\left[U_{\wedge}(\mathbf{s})+A_{\wedge}(\mathbf{s})\right] \wedge F_{\wedge}(\mathbf{s}) .
$$

Since $U_{\wedge}(\mathbf{s})=G(\mathbf{s})+F_{\wedge}(\mathbf{s})$, assuming that $F_{\wedge}(\mathbf{s})$ is finite yields

$$
G(\mathbf{s})=\min \left[0, G(\mathbf{s})+A_{\wedge}(\mathbf{s})\right] .
$$


The largest nontrivial solution of Eq. (10.24) is [Mar96]

$$
G(\mathbf{s})= \begin{cases}0 & \text { for } \mathbf{s} \in P, \\ -\infty & \text { for } \mathbf{s} \notin P,\end{cases}
$$

where $P$ is the convex planar region,

$$
P=\left\{\left(s_{1}, s_{2}\right): i s_{1}+j s_{2} \leq a_{i j} \forall(i, j) \in M_{0}\right\} .
$$

Thus, the system acts as an ideal-cutoff spatial slope filter, passing all input lower slope vectors $\mathbf{s}$ in the planar region $P$ unchanged but rejecting the rest. The inverse slope transform on $G$ yields the lower envelope $\breve{g}$ of the impulse response $g$. Over short-scale periods, $g$ has the shape induced by the sequence $\left\{a_{i j}\right\}$, but over scales much longer than the size of the output coefficient mask $M_{0}, g$ behaves like its lower envelope $\breve{g}$ (see the $1 \mathrm{D}$ example of Fig. 10.4b). Together $G$ and $\breve{g}$ can describe the long-scale dynamics of the system. In addition, if $g=\breve{g}$, then the above analysis is also exact for the short-scale behavior.

As an example let $M_{0}=\{(0,1),(0,1)\}$ and consider the min-sum difference equation,

$$
u(i, j)=\min \left(u(i-1, j)+a_{10}, u(i, j-1)+a_{01}, f(i, j)\right),
$$

which can model the forward pass of the sequential computation of the cityblock distance transform. Assuming boundary conditions $u(i, j)=+\infty$ if $i<0$ or $j<0$ and a bottom-left to top-right scanning order, the impulse response (found by induction) and slope response (shown in Fig. 10.5a for $a_{10}=a_{01}=1$ ) are

$$
\begin{aligned}
g(i, j) & =a_{10} i+a_{01} j-\zeta(i, j), \\
G\left(s_{1}, s_{2}\right) & =\zeta\left(a_{10}-s_{1}, a_{01}-s_{2}\right) .
\end{aligned}
$$

Thus, this system acts as a 2D lowpass spatial slope filter, passing all input lower slopes $s_{1} \leq a_{10}$ and $s_{2} \leq a_{01}$, but rejecting the rest. In this case, $g=\breve{g}$ is convex. This example demonstrates that the impulse response of ETI systems described by min-sum difference equations with a recursive part has an infinite support.

\subsection{PDEs for Morphological Image Analysis}

\subsubsection{PDEs Generating Dilations and Erosions}

Let $k: \mathbf{R}^{2} \rightarrow \overline{\mathbf{R}}$ be a unit-scale upper-semicontinuous concave structuring function, to be used as the kernel for morphological dilations and erosions. Scaling both its values and its support by a scale parameter $t \geq 0$ yields a parameterized family of multiscale structuring functions:

$$
k_{t}(x, y) \triangleq \begin{cases}t k(x / t, y / t) & \text { for } t>0 \\ \xi(0,0), & \text { for } t=0\end{cases}
$$




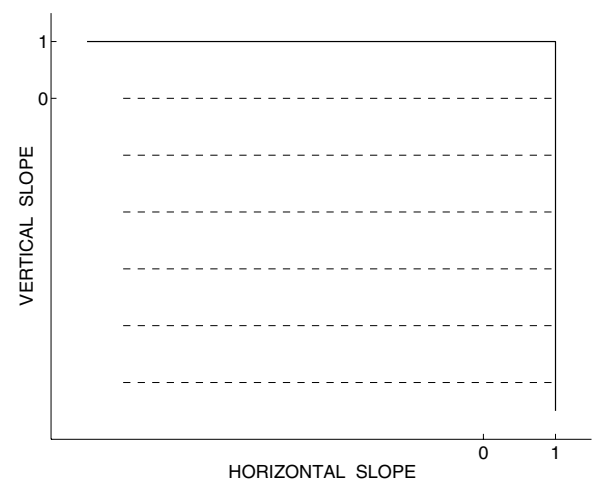

(a)

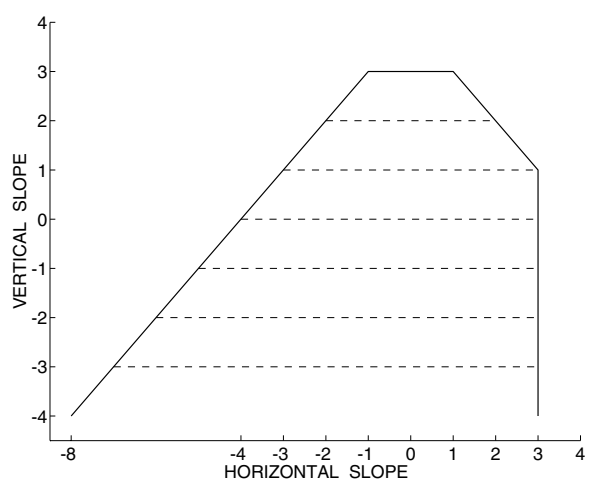

(b)

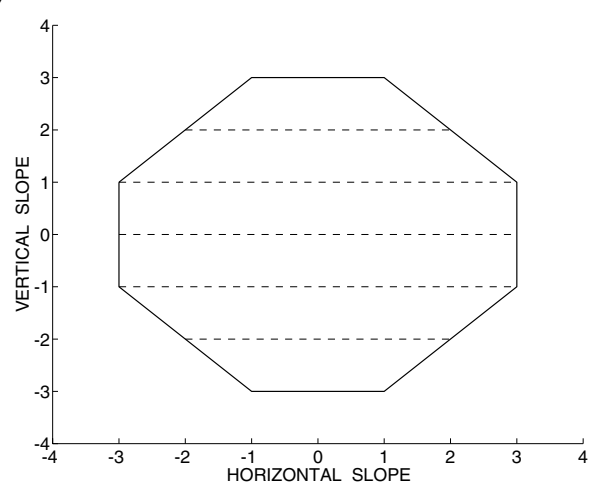

(c)

Figure 10.5: Regions of support of binary slope responses of discrete ETI systems, representing: (a) the forward pass of the cityblock distance transform, (b) the forward pass of the chamfer $(3,4)$ distance transform, and (c) the chamfer $(3,4)$ distance transform.

which satisfies the semigroup property $k_{s} \oplus k_{t}=k_{s+t}$. Using $k_{t}$ in place of $g$ as the kernel in the basic morphological operations leads to defining the multiscale dilation and erosion of $f: \mathbf{R}^{2} \rightarrow \mathbf{R}$ by $k_{t}$ as the scale-space functions

$$
\delta(x, y, t) \triangleq f \oplus k_{t}(x, y), \quad \varepsilon(x, y, t) \triangleq f \ominus k_{t}(x, y),
$$

where $\delta(x, y, 0)=\varepsilon(x, y, 0)=f(x, y)$.

In practice, a useful class of functions $k$ consists of flat structuring functions,

$$
k(x, y)= \begin{cases}0 & \text { for }(x, y) \in B \\ -\infty & \text { for }(x, y) \notin B\end{cases}
$$

that are the $0 /-\infty$ indicator functions of compact convex planar sets $B$. The general PDE generating the multiscale flat dilations of $f$ by a general compact convex symmetric $B$ is [Alv93, Bro94, Hei97]

$$
\delta_{t}=\operatorname{sptf}(B)\left(\delta_{x}, \delta_{y}\right),
$$


where $\operatorname{sptf}(B)$ is the support function of $B$,

$$
\operatorname{sptf}(B)(x, y) \triangleq \bigvee_{(a, b) \in B} a x+b y .
$$

Useful cases of structuring sets $B$ are obtained by the unit balls

$$
B_{p}=\left\{(x, y) \in \mathbf{R}^{2}:\|(x, y)\|_{p} \leq 1\right\}
$$

of the metrics induced by the $L_{p}$ norms $\|\cdot\|_{p}$, for $p=1,2, \ldots, \infty$. The PDEs generating the multiscale flat dilations of $f$ by $B_{p}$ for three special cases of norms $\|\cdot\|_{p}$ are as follows:

$$
\begin{aligned}
& B=\operatorname{rhombus}(p=1) \Longrightarrow \delta_{t}=\max \left(\left|\delta_{x}\right|,\left|\delta_{y}\right|\right)=\|\nabla \delta\|_{\infty} \text {, } \\
& B=\operatorname{disk}(p=2) \Longrightarrow \delta_{t}=\sqrt{\left(\delta_{x}\right)^{2}+\left(\delta_{y}\right)^{2}}=\|\nabla \delta\|_{2}, \\
& B=\text { square }(p=\infty) \Longrightarrow \delta_{t}=\left|\delta_{x}\right|+\left|\delta_{y}\right|=\|\nabla \delta\|_{1} \text {, }
\end{aligned}
$$

with $\delta(x, y, 0)=f(x, y)$. The corresponding PDEs generating mutliscale flat erosions are

$$
\begin{aligned}
B=\text { rhombus } & \Longrightarrow \varepsilon_{t}=-\|\nabla \varepsilon\|_{\infty}, \\
B=\text { disk } & \Longrightarrow \varepsilon_{t}=-\|\nabla \varepsilon\|_{2}, \\
B=\text { square } & \Longrightarrow \varepsilon_{t}=-\|\nabla \varepsilon\|_{1},
\end{aligned}
$$

with $\varepsilon(x, y, 0)=f(x, y)$.

These simple but nonlinear PDEs are satisfied at points where the data are smooth, that is, the partial derivatives exist. However, even if the initial image or signal $f$ is smooth, at finite scales $t>0$ the above dilation or erosion evolution may create discontinuities in the derivatives, called shocks, which then continue propagating in scale-space. Thus, the multiscale dilations $\delta$ or erosions $\varepsilon$ are weak solutions of the corresponding PDEs, in the sense put forth by Lax [Lax73]. Ways to deal with these shocks include replacing standard derivatives with morphological derivatives [Bro94] or replacing the PDEs with differential inclusions [Mat93]. For example, let

$$
\mathcal{M}_{x} f(x, y) \triangleq \lim _{r \downarrow 0} \frac{\sup \{f(x+v, y):|v| \leq r\}-f(x, y)}{r}
$$

be the sup-derivative of $f$ along the $x$-direction. If the right $\left[f_{x}(x+, y)\right]$ and left $\left[f_{x}(x-, y)\right]$ derivatives of $f$ along the $x$-direction exist, then

$$
\mathcal{M}_{x} f(x, y)=\max \left[0, f_{x}(x+, y),-f_{x}(x-, y)\right] .
$$

The sup-derivative $\mathcal{M}_{y} f$ along the $y$-direction can be treated similarly. Then, a generalized PDE generating flat dilations by a compact convex symmetric $B$ is

$$
\delta_{t}=\operatorname{sptf}(B)\left(\mathcal{M}_{x} \delta, \mathcal{M}_{y} \delta\right)
$$


This new PDE can handle discontinuities (i.e., shocks) in the partial derivatives of $\delta$, provided that its left and right derivatives exist everywhere.

The above PDEs for dilations-erosions of graylevel images by flat structuring elements directly apply to binary images, since flat dilations-erosions commute with thresholding, and hence when the graylevel image is dilated-eroded, each of its thresholded versions representing a binary image is simultaneously dilatederoded by the same element and at the same scale. However, this is not the case with graylevel structuring functions. We provide two examples of PDEs generating multiscale dilations by graylevel structuring functions: If $k$ is the compact-support spherical function

$$
k(x, y)= \begin{cases}\sqrt{1+x^{2}+y^{2}} & \text { for } x^{2}+y^{2} \leq 1, \\ -\infty & \text { for } x^{2}+y^{2}>1,\end{cases}
$$

the dilation PDE becomes

$$
\delta_{t}=\sqrt{1+\left(\delta_{x}\right)^{2}+\left(\delta_{y}\right)^{2}} .
$$

For the infinite-support parabolic structuring function

$$
k(x, y)=-r\left(x^{2}+y^{2}\right), \quad r>0,
$$

the dilation PDE becomes

$$
\delta_{t}=\left[\left(\delta_{x}\right)^{2}+\left(\delta_{y}\right)^{2}\right] / 4 r .
$$

\subsubsection{Slope Transforms and Dilation PDEs}

All of the above dilation (and erosion) PDEs can be unified using slope transforms. Specifically, let the unit-scale kernel $k(x, y)$ be a general upper-semicontinuous concave function and consider its upper slope transform,

$$
K_{\vee}\left(s_{1}, s_{2}\right) \triangleq \bigvee_{(x, y) \in \mathbf{R}^{2}} k(x, y)-\left(s_{1} x+s_{2} y\right)
$$

Then, as discussed elsewhere [Hei97, Mat93], the PDE generating multiscale signal dilations by $k$ is

$$
\delta_{t}=K_{\vee}\left(\delta_{x}, \delta_{y}\right)
$$

Thus, the rate of change of $\delta$ in the scale $(t)$ direction is equal to the upper slope transform of the structuring function evaluated at the spatial gradient of $\delta$. Similarly, the PDE generating the multiscale erosion by $k$ is

$$
\varepsilon_{t}=-K_{\vee}\left(\varepsilon_{x}, \varepsilon_{y}\right) .
$$

All of the dilation and erosion PDEs examined are special cases of HamiltonJacobi equations, which are of paramount importance in physics. Such equations usually do not admit classic (i.e., everywhere differentiable) solutions. Viscosity 
solutions of Hamilton-Jacobi PDEs have been extensively studied by Crandall et al. [Cra92]. Based on the theory of viscosity solutions, Heijmans and Maragos [Hei97] have shown via slope transforms that the multiscale dilation by a general uppersemicontinuous concave function is the viscosity solution of the Hamilton-Jacobi dilation PDE of Eq. (10.47).

\subsubsection{Numerical Algorithm for Dilation PDEs}

The PDEs generating flat dilation and erosion by disks are special cases of HamiltonJacobi PDEs of the type

$$
\phi_{t}=\beta\|\nabla \phi\|_{2}
$$

where $\phi=\phi(x, y, t)$ and where $\beta=\beta(x, y)$ has constant sign for all $(x, y)$. An efficient algorithm for numerically solving such PDEs for applications of curve evolution has been developed by Osher and Sethian [Osh88] by adapting the technology of conservative monotone discretization schemes for shock-producing PDEs of hyperbolic conservation laws [Lax73]. The main steps of such a first-order algorithm are

$$
\begin{aligned}
\Phi_{i, j}^{n}= & \text { approximation of } \phi(i \Delta x, j \Delta y, n \Delta t) \text { on a grid, } \\
V_{i j}= & \beta(i \Delta x, j \Delta y), \\
D_{+x} \Phi_{i, j}^{n}= & \left(\Phi_{i+1, j}^{n}-\Phi_{i, j}^{n}\right) / \Delta x, \quad D_{-x} \Phi_{i, j}^{n}=\left(\Phi_{i, j}^{n}-\Phi_{i-1, j}^{n}\right) / \Delta x \\
D_{+y} \Phi_{i, j}^{n}= & \left(\Phi_{i, j+1}^{n}-\Phi_{i, j}^{n}\right) / \Delta y, \quad D_{-y} \Phi_{i, j}^{n}=\left(\Phi_{i, j}^{n}-\Phi_{i, j-1}^{n}\right) / \Delta y, \\
H= & {\left[\max \left(0, D_{-x} \Phi_{i, j}^{n}\right)\right]^{2}+\left[\min \left(0, D_{+x} \Phi_{i, j}^{n}\right)\right]^{2}+\left[\max \left(0, D_{-y} \Phi_{i, j}^{n}\right)\right]^{2} } \\
& +\left[\min \left(0, D_{+y} \Phi_{i, j}^{n}\right)\right]^{2}, \\
\Phi_{i, j}^{n+1}= & \Phi_{i, j}^{n}+\Delta t V_{i j} \sqrt{H} \quad n=0,1,2, \ldots,\left(T_{\max } / \Delta t\right),
\end{aligned}
$$

where $T_{\max }$ is the maximum time (or scale) of interest, $\Delta x$, and $\Delta y$ are the spatial grid spacings, and $\Delta t$ is the time (scale) step. For stability, the space/time steps must satisfy $(\Delta t / \Delta x+\Delta t / \Delta y) V_{i j} \leq 0.5$.

By choosing fine grids (and possibly higher-order terms) an arbitrarily low error (between signal values on the continuous plane and the discrete grid) can be achieved in implementing morphological operations involving disks as structuring elements. This is a significant advantage of the PDE approach, as observed elsewhere [Are93, Sap93]. Thus, curve evolution provides a geometrically better implementation of multiscale morphological operations with the disk-shaped structuring element. Figure 10.6 shows the results of a simulation to compare the traditional dilation of digital images via discrete max-sum convolution of the image by digital approximations to disks (e.g., squares) versus a dilation that is the solution $\delta(x, y, t)$ of the dilation PDE numerically solved using algorithm (10.49). Comparing the graylevel images to their binary versions (from thresholding at level $=0$ ), it is evident that the PDE approach to multiscale dilations can give much better approximations to Euclidean disks and hence avoid the abrupt shape discretization inherent in modeling digital multiscale dilations using discrete disks. 


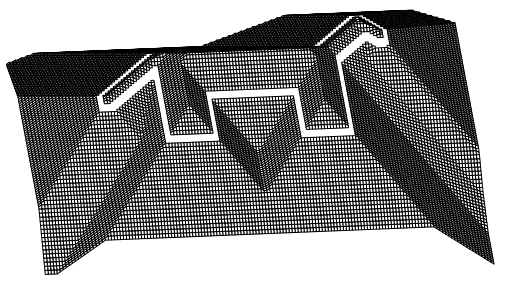

(a)

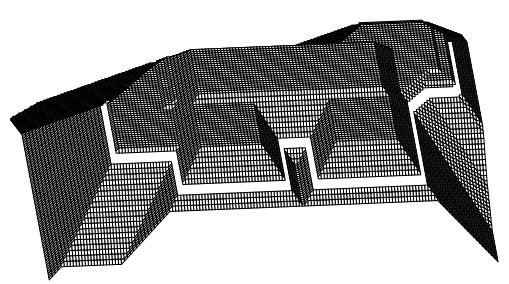

(c)

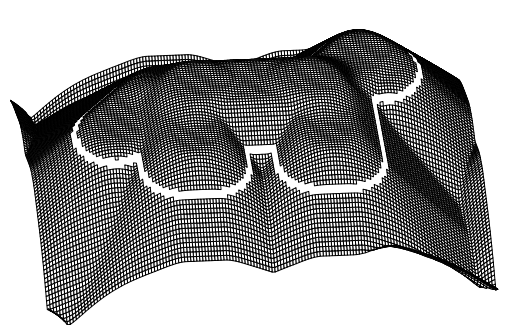

(e)

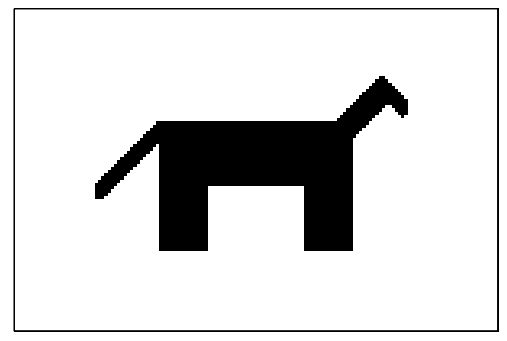

(b)

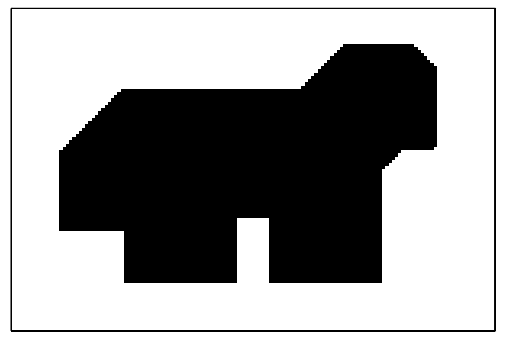

(d)

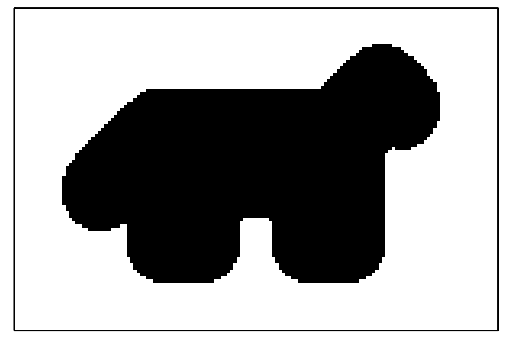

(f)

Figure 10.6: (a) Original digital graylevel image $f$ and its contour at level $=0$. (b) Binary image $S$ from thresholding $f$ at level $=0$. (c) Flat dilation $f \oplus B$ of $f$ by a discrete disk, that is, a square of $(2 t+1) \times(2 t+1)$ pixels, with $t=5$. (d) Binary image $S \oplus B$ from thresholding $f \oplus B$ at level $=0$. (e) Dilation of $f$ by running the PDE $\partial \delta / \partial t=\|\nabla \delta\|_{2}$ for scales $t \in[0,5]$ with initial condition $\delta(x, y, 0)=f(x, y)$. (f) Binary image from thresholding the image in (e) at level $=0$.

\subsubsection{PDEs Generating Openings and Closings}

Let $u(x, y, t)=[f(x, y) \ominus t B] \oplus t B$ be the multiscale flat opening of an image $f$ by the disk $B$. This standard opening can be generated at any scale $r>0$ by running the following PDE [Alv93]

$$
u_{t}=-\max (\operatorname{sgn}(r-t), 0)\|\nabla u\|_{2}+\max (\operatorname{sgn}(t-r), 0)\|\nabla u\|_{2},
$$

from time $t=0$ until time $t=2 r$ with initial condition $u(x, y, 0)=f(x, y)$, where $\operatorname{sgn}(\cdot)$ denotes the signum function. This PDE has time-dependent switching coefficients that make it act as an erosion PDE during $t \in[0, r]$ but as a dilation PDE during $t \in[r, 2 r]$. The discontinuities that this PDE exhibits at the instants 
it switches can be dealt with by making appropriate changes to the time scale, as suggested by Alvarez et al. [Alv93].

Recently, the reconstruction openings have found many more applications than the standard openings in a large variety of problems. We next present a nonlinear PDE, introduced by Maragos and Meyer [Mar99], that can model and generate openings and closings by reconstruction. Consider a 2D reference signal $f(x, y)$ and a marker signal $g(x, y)$. If $g \leq f$ everywhere and we start iteratively growing $g$ via incremental flat dilations with an infinitesimally small disk $\Delta t B$ but without ever growing the result above the graph of $f$, then in the limit we shall have produced the reconstruction opening of $f$ (with respect to the marker $g$ ). The infinitesimal generator of this signal evolution can be modeled via a dilation PDE that has a mechanism to stop the growth whenever the intermediate result attempts to create a function larger than $f$. Specifically, let $u(x, y, t)$ represent the evolutions of $f$ with initial value $u_{0}(x, y)=u(x, y, 0)=g(x, y)$. Then, $u$ is a weak solution of the PDE

$$
\begin{aligned}
u_{t}(x, y, t) & =\|\nabla u(x, y, t)\| \operatorname{sgn}[f(x, y)-u(x, y, t)] \\
u(x, y, 0) & =g(x, y) .
\end{aligned}
$$

This PDE models a conditional dilation that grows the intermediate result as long as it does not exceed $f$. In the limit we obtain the final result $u_{\infty}(x, y)=\lim _{t \rightarrow \infty}$ $u(x, y, t)$. The mapping $u_{0} \mapsto u_{\infty}$ is the reconstruction opening filter.

If in the PDE of (10.51) we reverse the order between $f$ and $g$ (i.e., assume that $g \geq f$ ), then the positive growth (dilation) of $g$ is replaced with negative growth (erosion) because now $\operatorname{sgn}(f-u) \leq 0$. This negative growth stops when the intermediate result attempts to become smaller than $f$; in the limit we obtain the reconstruction closing of $f$ with respect to the marker $g$.

The following shock-capturing and entropy-satisfying numerical algorithm has been used to solve the PDE of (10.51) in [Mar99]. Let $U_{i, j}^{n}$ be the approximation of $u(x, y, t)$ on a computational grid $(i \Delta x, j \Delta y, n \Delta t)$. We then approximate PDE (10.51) by the following 2D nonlinear difference equation:

$$
\begin{aligned}
& U_{i, j}^{n+1}=U_{i, j}^{n}-\Delta t[\cdots \\
& \left(S_{i, j}^{n}\right)^{+} \sqrt{\left(\left(D_{-x} U_{i, j}^{n}\right)^{+}\right)^{2}+\left(\left(D_{+x} U_{i, j}^{n}\right)^{-}\right)^{2}+\left(\left(D_{-y} U_{i, j}^{n}\right)^{+}\right)^{2}+\left(\left(D_{+y} U_{i, j}^{n}\right)^{-}\right)^{2}}+ \\
& \left.\left(S_{i, j}^{n}\right)^{-} \sqrt{\left(\left(D_{+x} U_{i, j}^{n}\right)^{+}\right)^{2}+\left(\left(D_{-x} U_{i, j}^{n}\right)^{-}\right)^{2}+\left(\left(D_{+y} U_{i, j}^{n}\right)^{+}\right)^{2}+\left(\left(D_{-y} U_{i, j}^{n}\right)^{-}\right)^{2}}\right],
\end{aligned}
$$

where $S_{i, j}^{n}=\operatorname{sgn}\left(f(i \Delta x, j \Delta y)-U_{i, j}^{n}\right)$ and we denote $r^{+} \triangleq \max (r, 0), r^{-} \triangleq$ $\min (r, 0)$. For stability, $(\Delta t / \Delta x+\Delta t / \Delta y) \leq 0.5$ is required. Also, a sign consistency is enforced at each iteration: $\operatorname{sgn}\left(U^{n}-f\right)=\operatorname{sgn}(g-f)$. Examples of simulating this numerical algorithm are shown in Fig. 10.7 .

What happens if we now use the PDE of (10.51) when there is no specific order between $f$ and $g$ ? In such a case, the PDE has a sign-varying coefficient $\operatorname{sgn}(f-u)$ with spatiotemporal dependence, which controls the instantaneous growth and stops it whenever $f=u$. (Of course, there also is no growth at stationary points, 


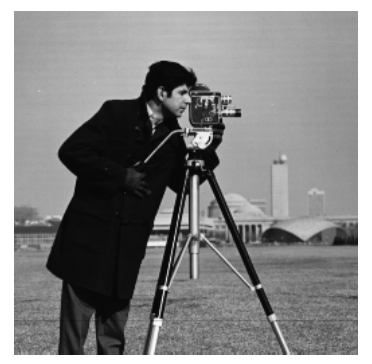

(a)

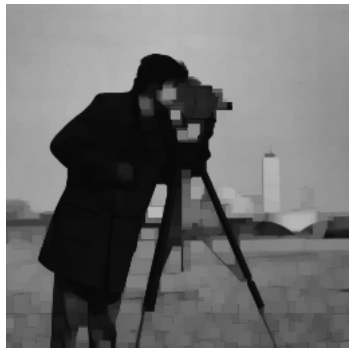

(b)

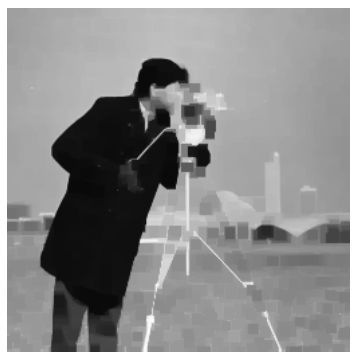

(e)

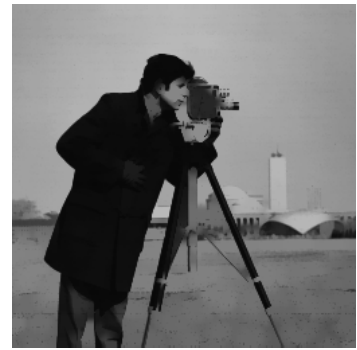

(c)

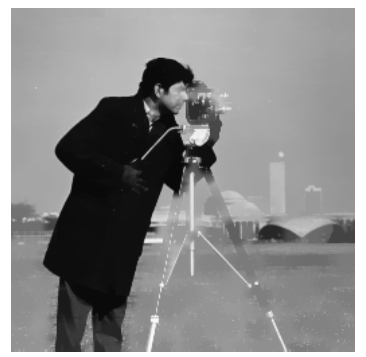

(f)

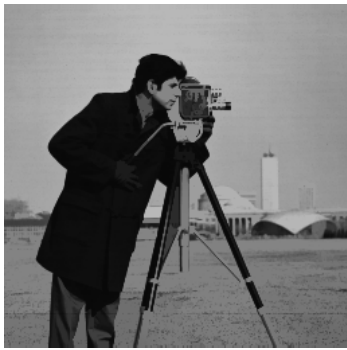

(d)

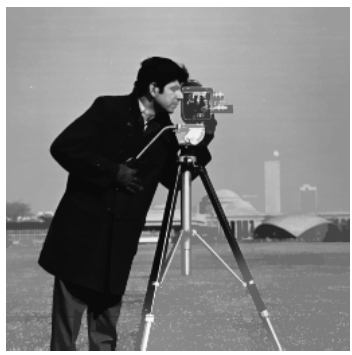

(g)

Figure 10.7: Morphological reconstruction filtering generated by the PDE (10.51) using two markers. (a) Reference image Cameraman, $256 \times 256$ pixels. Second row: (b) the marker $(t=0)$ was a standard opening by a $7 \times 7$ square of pixels, (c) the evolution at $t=20 \Delta t$, and (d) the final reconstruction opening (after convergence). Third row: (e) the marker $(t=0)$ was a standard closing by a square of $7 \times 7$ pixels, (f) the evolution at $t=20 \Delta t$, and (g) the final reconstruction closing (after convergence). $(\Delta x=\Delta y=1, \Delta t=0.25$.)

where $u_{x}=u_{y}=0$.) The control mechanism is of a switching type: For each $t$, at points $(x, y)$ where $u(x, y, t)<f(x, y)$, it acts as a dilation PDE, whereas if $u(x, y, t)>f(x, y)$, it acts as an erosion PDE and reverses the direction of propagation. The final result $u_{\infty}(x, y)=\lim _{t \rightarrow \infty} u(x, y, t)$ is equal to the output from a general class of morphological filters, called levelings, which were introduced by Meyer [Mey98], have many useful properties, and contain as special cases the reconstruction openings and closings. 


\subsection{Curve Evolution}

Consider at time $t=0$ an initial simple, smooth, closed planar curve $\gamma(0)$ that is propagated along its normal vector field at speed $V$ for $t>0$. Let this evolving curve (front) $\gamma(t)$ be represented by its position vector $\vec{C}(p, t)=(x(p, t), y(p, t))$ and be parameterized by $p \in[0, J]$ so that it has its interior on the left in the direction of increasing $p$ and $\vec{C}(0, t)=\vec{C}(J, t)$. The curvature along the curve is

$$
\kappa=\kappa(p, t) \triangleq \frac{y_{p p} x_{p}-y_{p} x_{p p}}{\left(x_{p}^{2}+y_{p}^{2}\right)^{3 / 2}} .
$$

A general front propagation law (flow) is

$$
\frac{\partial \vec{C}(p, t)}{\partial t}=V \vec{N}(p, t),
$$

with initial condition $\gamma(0)=\{\vec{C}(p, 0): p \in J\}$, where $\vec{N}(p, t)$ is the instantaneous unit outward normal vector at points on the evolving curve and $V=\vec{C}_{t} \cdot \vec{N}$ is the normal speed, with $\vec{C}_{t}=\partial \vec{C} / \partial t$. This speed may depend on local geometrical information such as the curvature, global image properties, or other factors independent of the curve. If $V=1$ or $V=-1$, then $\gamma(t)$ is the boundary of the dilation or erosion of the initial curve $\gamma(0)$ by a disk of radius $t$. In general, if $B$ is an arbitrary compact, convex, symmetric planar set of unit scale and if we dilate the initial curve $\gamma(0)$ with $t B$ and set the new curve $\gamma(t)$ equal to the outward boundary of $\gamma(0) \oplus t B$, then this curve evolution can also be generated by the PDE of Eq. (10.54) using a speed [Are93, Sap93]

$$
V=\operatorname{sptf}(B)(\vec{N}),
$$

where $\operatorname{sptf}(B)$ is the support function of $B$.

Another important speed model has been studied extensively by Osher and Sethian [Osh88, Set96] for general evolution of interfaces and by Kimia et al. [Kim90] for shape analysis in computer vision:

$$
V=1-\epsilon \kappa, \quad \epsilon \geq 0 .
$$

As analyzed by Sethian [Set96], when $V=1$ the front's curvature will develop singularities, and the front will develop corners (i.e., the curve derivatives will develop shocks-discontinuities) at finite time if the initial curvature is anywhere negative. Two ways to continue the front beyond the corners are as follows: (1) If the front is viewed as a geometric curve, then each point is advanced along the normal by a distance $t$, and hence a "swallowtail" is formed beyond the corners by allowing the front to pass through itself. 2) If the front is viewed as the boundary separating two regions, an entropy condition is imposed to disallow the front to pass through itself. In other words, if the front is a propagating flame, then "once a particle is burnt it stays burnt" [Set96]. The same idea has also been used to model grassfire propagation leading to the medial axis of a shape [Blu73]. It is 
equivalent to using Huygen's principle to construct the front as the set of points at distance $t$ from the initial front. This can also be obtained from multiscale dilations of the initial front by disks of radii $t>0$. Both the swallowtail and the entropy solutions are weak solutions. The examples in Fig. 10.8 show that, when $\epsilon>0$, motion with curvature-dependent speed has a smoothing effect. Further, the limit of the solution for the $V=1-\epsilon \kappa$ case as $\epsilon \downarrow 0$ is the entropy solution for the $V=1$ case [Set96].

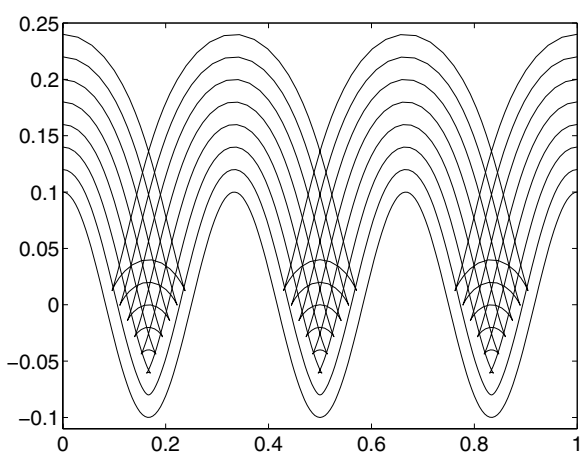

(a)

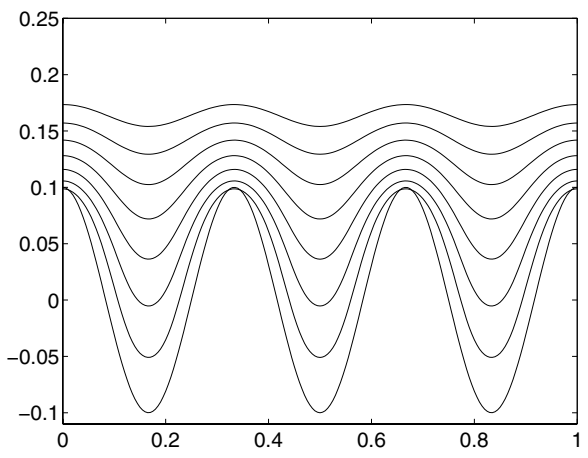

(c)

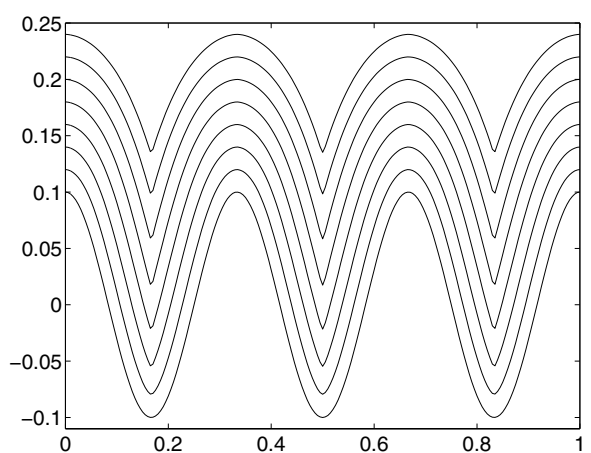

(b)

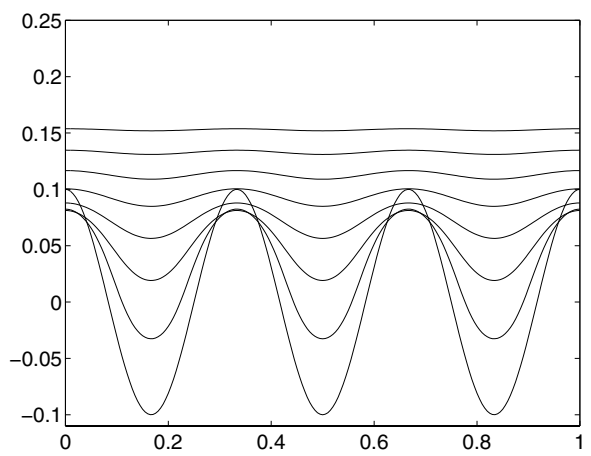

(d)

Figure 10.8: Evolution of the curve (signal graph) $(-p, \cos (6 \pi p) / 10), p \in[0,1]$. Evolved curves are plotted from $t=0$ to $t=0.14$ at increments of 0.02 . The numerical simulation for (b), (c), and (d) is based on the Osher and Sethian algorithm with $\Delta x=0.005$ and $\Delta t$ chosen small enough for stability. (a) $V=1$, "swallowtail" weak solution. (b) $V=1$, entropy weak solution with $\Delta t=0.002$. (c) $V=1-0.05 \kappa$ with $\Delta t=0.0002$. (d) $V=1-0.1 \kappa$ with $\Delta t=0.0001$.

To overcome the topological problem of splitting and merging and numerical problems with the Lagrangian formulation of Eq. (10.54), an Eulerian formulation was proposed by Osher and Sethian [Osh88] in which the original curve $\gamma(0)$ is first embedded in the surface of an arbitrary 2D Lipschitz continuous function $\phi_{0}(x, y)$ as its level set (contour line) at zero level. For example, we can select $\phi_{0}(x, y)$ to be equal to the signed distance function from the boundary of $\gamma(0)$, positive (negative) in the interior (exterior) of $\gamma(0)$. Then, the evolving planar curve 
is embedded as the zero-level set of an evolving space-time function $\phi(x, y, t)$ :

$$
\begin{aligned}
& \gamma(t)=\{(x, y): \phi(x, y, t)=0\} \\
& \gamma(0)=\left\{(x, y): \phi_{0}(x, y, 0)=\phi(x, y)=0\right\} .
\end{aligned}
$$

Geometrical properties of the evolving curve can be obtained from spatial derivatives of the level function. Thus, at any point on the front the curvature and outward normal of the level sets can be found from $\phi$ :

$$
\vec{N}=-\frac{\nabla \phi}{\|\nabla \phi\|}, \quad \kappa=-\operatorname{div}\left(\frac{\nabla \phi}{\|\nabla \phi\|}\right) .
$$

The curve evolution PDE of Eq. (10.54) induces a PDE generating its level function:

$$
\begin{aligned}
\phi_{t} & =V\|\nabla \phi\|, \\
\phi(x, y, 0) & =\phi_{0}(x, y) .
\end{aligned}
$$

If $V=1$, the above function evolution PDE is identical to the flat circular dilation PDE of Eq. (10.35) by equating scale with time. Thus, we can view this specific dilation PDE as a special case of the general function evolution PDE of Eq. (10.60) in which all level sets expand in a homogeneous medium with $V=1$. Propagation in a heterogeneous medium with $V=V(x, y)>0$ will lead later to the eikonal PDE.

\subsection{Distance Transforms}

\subsubsection{Distance Transforms and Wave Propagation}

For binary images, the distance transform is a compact way to represent their multiscale dilations and erosions by convex polygonal structuring elements whose shape depends upon the norm used to measure distances. Specifically, a binary image can be divided into the foreground set $S \subseteq \mathbf{R}^{2}$ and the background set $S^{c}=\{(x, y):(x, y) \notin S\}$. For shape analysis of an image object $S$, it is often more useful to consider its inner distance transform by using $S$ as the domain to measure distances from its background. However, for the applications discussed herein, we need to view $S$ as a region marker or a source emanating a wave that will propagate away from it into the domain of $S^{c}$. Thus, we define the outer distance transform of a set $S$ with respect to the metric induced by some norm $\|\cdot\|_{p}$, $p=1,2, \ldots, \infty$, as the distance function:

$$
\mathrm{D}_{p}(S)(x, y) \triangleq \bigwedge_{(v, w) \in S}\|(x-v, y-w)\|_{p} .
$$

If $B_{p}$ is the unit ball induced by the norm $\|\cdot\|_{p}$, thresholding the distance transform at level $r>0$ and obtaining the corresponding level set yields the morphological dilation of $S$ by the ball $B_{p}$ at scale $r$ :

$$
S \oplus r B_{p}=\left\{(x, y): \mathrm{D}_{p}(S)(x, y) \geq r\right\} .
$$


The boundaries of these dilations are the wavefronts of the distance propagation. Multiscale erosions of $S$ can be obtained from the outer distance transform of $S^{c}$.

In addition to being a compact representation for multiscale erosions and dilations, the distance transform has found many applications in image analysis and computer vision. Examples include smoothing, skeletonization, size distributions, shape description, object detection and recognition, segmentation, and path finding [Blu73, Bor86, Nac96, Pre93, Ros66, Ros68, Ver91, Vin91b]. Thus, many algorithms have been developed for its computation.

Using Huygen's construction, the boundaries of multiscale dilations-erosions by disks can also be viewed as the wavefronts of a wave initiating from the original image boundary and propagating with constant normal speed, that is, in a homogeneous medium. Thus, the distance function has a minimum time-of-arrival interpretation [Blu73], and its isolevel contours coincide with those of the wave phase function. Points at which these wavefronts intersect and extinguish themselves (according to Blum's grassfire propagation principle) are the points of the Euclidean skeleton axis of $S$ [Blu73]. Overall, the Euclidean distance function $\mathrm{D}_{2}(S)$ is the weak solution of the following nonlinear PDE:

$$
\begin{aligned}
\|\nabla E(x, y)\|_{2}=1, & (x, y) \in S^{c}, \\
E(x, y)=0, & (x, y) \in \partial S .
\end{aligned}
$$

This is a special case of the eikonal PDE $\|\nabla E(x, y)\|_{2}=\eta(x, y)$ that corresponds to wave propagation in heterogeneous media and whose solution $E$ is a weighted distance function, whose weights $\eta(x, y)$ are inversely proportional to the varying propagation speed [Lev70, Rou92, Ver90].

\subsubsection{Distance Transforms as Infimal Convolutions and Slope Filters}

If we consider the $0 / \infty$ indicator function of $S$,

$$
\mathrm{I}(S)(x, y) \triangleq \begin{cases}0 & \text { for }(x, y) \in S, \\ +\infty & \text { for }(x, y) \notin S\end{cases}
$$

and the $L_{p}$ norm structuring function,

$$
g_{p}(x, y)=\|(x, y)\|_{p},
$$

it follows that the distance transform can be obtained from the infimal convolution of the indicator function of the set with the norm function:

$$
\mathrm{D}_{p}(S)=\mathrm{I}(S) \oplus^{\prime} g_{p}
$$

Further, since the relative ordering of distance values does not change if we raise them to a positive power $m>0$, it follows that we can obtain powers of the distance function by convolving with the respective powers of the norm function:

$$
\left[\mathrm{D}_{p}(S)\right]^{m}=\mathrm{I}(S) \oplus^{\prime}\left(g_{p}\right)^{m} .
$$


The infimal convolution in Eq. (10.66) is equivalent to passing the input signal, that is, the set's indicator function, through an ETI system with slope response [Mar96]

$$
G_{\wedge}(\mathbf{s})= \begin{cases}0 & \text { for }\|\mathbf{s}\|_{q} \leq 1, \\ -\infty & \text { for }\|\mathbf{s}\|_{q}>1,\end{cases}
$$

where $q$ is the conjugate exponent of $p(1 / p+1 / q=1)$. That is, the distance transform is the output of an ideal-cutoff slope-selective filter that rejects all input planes whose slope vector falls outside the unit ball with respect to the $\|\cdot\|_{q}$ norm but passes all the rest unchanged.

\subsubsection{Euclidean Distance Transforms of Binary Images and Approximations}

To obtain isotropic distance propagation, we want to employ the Euclidean distance transform, that is, using the norm $\|\cdot\|_{2}$ in Eq. (10.61), since it gives multiscale morphology with the disk as the structuring element. However, computing the Euclidean distance transform of discrete images has a significant computational complexity. Thus, various techniques have been used to obtain an approximate or the exact Euclidean distance transform at a lower complexity. Four types of approaches that deal with this problem are as follows:

(1) Discrete metrics on grids that yield approximations to the Euclidean distance. Their early theory was developed by Rosenfeld and Pfaltz [Ros66, Ros68], based on either sequential or parallel operations. This was followed later by a generalization developed by Borgefors [Bor86] and based on chamfer metrics that yielded improved approximations to the Euclidean distance.

(2) Fast algorithmic techniques that can obtain the exact Euclidean distances by operating on complex data structures (e.g., [Dan80, Vin91a]).

(3) Infimal convolutions of binary images with a parabolic structuring function, which yield the exact squared Euclidean distance transform [Ste80, Boo92, Hua94]. This follows from Eq. (10.67) by using $m=2$ with the Euclidean norm $(p=2)$ :

$$
\left[\mathrm{D}_{2}(S)\right]^{2}=\mathrm{I}(S) \oplus^{\prime}\left(g_{2}\right)^{2} .
$$

The kernel in the above infimal convolution is a convex parabola $\left[g_{2}(x, y)\right]^{2}=$ $\|(x, y)\|_{2}^{2}=x^{2}+y^{2}$. Note that the above result holds for images and kernels defined both on the continuous and on the discrete plane. Of course, convolution of the image with an infinite-extent kernel is not possible, and hence truncation of the parabola is used, which incurs an approximation error. The complexity of this convolution approach can be reduced significantly by using dimensional decomposition of the 2D parabolic structuring function by expressing it either as the dilation of two 1D quadratic structuring functions [Boo92] or as the dilation of several $3 \times 3$ kernels that yields a truncation of the 2D parabola [Hua94, Shi91].

(4) Efficient numerical algorithms for solving the nonlinear PDE (10.63) that yield arbitrarily close approximations to the Euclidean distance function. 
Approach (4) yields the best approximations and will be discussed later. Of the other three approaches, (1) and (3) are more general than (2), have significant theoretical structure, and can be used with even the simplest data structures, such as rectangularly or hexagonally sampled image signals. Next we elaborate on approach (1), which has been studied the most.

\subsubsection{Chamfer Distance Transforms}

The general chamfer distance transform is obtained by propagating local distance steps within a small neighborhood. For each such neighborhood the distance steps form a mask of weights that is infimally convolved with the image. For a $3 \times 3$-pixel neighborhood, if $a$ and $b$ are the horizontal and diagonal distance steps, respectively, the outer $(a, b)$ chamfer distance transform of a planar set $S$ can also be obtained directly from the general definition in Eq. (10.61) by replacing the general $L_{p}$ norm $\|\cdot\|_{p}$ with the $(a, b)$ chamfer norm

$$
\|(x, y)\|_{a, b} \triangleq \max (|x|,|y|) a+\min (|x|,|y|)(b-a) .
$$

The unit ball corresponding to this chamfer norm is a symmetric octagon, and the resulting distance transform is

$$
\mathrm{D}_{a, b}(S)(x, y)=\bigwedge_{(v, w) \in S}\|(x-v, y-w)\|_{a, b} .
$$

Note that the above two equations apply to sets $S$ and points $(x, y)$ both in the continuous plane $\mathbf{R}^{2}$ as well as in the discrete plane $\mathbf{Z}^{2}$.

For a $3 \times 3$-pixel neighborhood, the outer $(a, b)$ chamfer distance transform of a discrete set $S \subseteq \mathbf{Z}^{2}$ can be obtained via the following sequential computation [Bor86, Ros66]:

$$
\begin{aligned}
u_{n}(i, j)= & \min \left(u_{n}(i-1, j)+a, u_{n}(i, j-1)+a,\right. \\
& \left.u_{n}(i-1, j-1)+b, u_{n}(i+1, j-1)+b, u_{n-1}(i, j)\right) .
\end{aligned}
$$

Starting from $u_{0}=\mathrm{I}(S)$ as the $0 / \infty$ indicator function of $S$, two passes $(n=1,2)$ of the 2D recursive erosion of Eq. (10.72) suffice to compute the chamfer distance transform of $S$ if $S^{c}$ is bounded and simply connected. During the first pass the image is scanned from top-left to bottom-right using the four-point nonsymmetric half-plane submask of the $3 \times 3$ neighborhood. During the second pass the image is scanned in the reverse direction using the reflected submask of distance steps. The final result $u_{2}(i, j)$ is the outer $(a, b)$ chamfer distance transform of $S$ evaluated at points of $\mathbf{Z}^{2}$. An example of the three images, $u_{0}, u_{1}$, and $u_{2}$, is shown in Fig. 10.9.

Thus, the sequential implementation of the local distance propagation is done via simple recursive min-sum difference equations. We shall show that these equations correspond to ETI systems with infinite impulse responses and binary slope responses. The distance propagation can also be implemented in parallel via nonrecursive min-sum equations, which correspond to ETI systems with finite impulse responses, as explained next. 


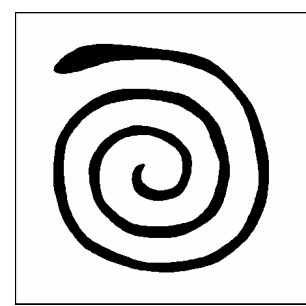

(a)

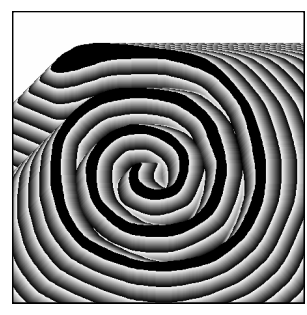

(b)

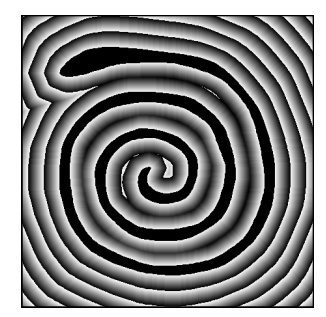

(c)

Figure 10.9: Sequential computation of the chamfer distance transform with optimal distance steps in a $3 \times 3$ mask. (a) Original binary image, $450 \times 450$ pixels, (b) result after forward scan, and (c) final result after backward scan. [In (b) and (c) the distances are displayed as intensity values modulo a constant.]

\section{Sequential Computation and IIR Slope Filters}

Consider a 2D min-sum autoregressive difference equation with output mask and coefficients as in Fig. 10.10c:

$$
\begin{aligned}
u(i, j)= & \min (u(i-1, j)+a, u(i, j-1)+a, \\
& u(i-1, j-1)+b, u(i+1, j-1)+b, f(i, j)),
\end{aligned}
$$

where $f=\mathrm{I}(S)$ is the $0 / \infty$ indicator function of a set $S$ representing a discrete binary image. Then consider the following distance transformation of $f$ obtained in two passes: During the forward pass Eq. (10.73) is recursively run over $f(i, j)$ in a bottom-to-top, left-to-right scanning order. The forward pass mapping $f \mapsto u$ is an ETI system with an infinite impulse response (found via induction):

$$
g_{f}(i, j)= \begin{cases}\|(i, j)\|_{a, b} & \text { for } i+j \geq 0, j \geq 0, \\ +\infty & \text { otherwise. }\end{cases}
$$

A truncated version of $g_{f}$ is shown in Fig. 10.10d. The slope response $G_{f}(\mathbf{s})$ of this ETI system is equal to the $0 /-\infty$ indicator function of the region shown (for $a=3, b=4$ ) in Fig. 10.5(b).

During the backward pass a recursion similar to Eq. (10.73) but in the opposite scanning order and using as output mask the reflected version of Fig. 10.10c is run over the previous result $u(i, j)$ to yield a signal $d(i, j)$ that is the final distance transform of $f(i, j)$. The backward pass mapping $u \mapsto d$ is an ETI system with an infinite impulse response $g_{b}(i, j)=g_{f}(-i,-j)$ and a slope response $G_{b}(\mathbf{s})=$ $G_{f}(-\mathbf{s})$.

Since infimal convolution is an associative operation, the distance transform mapping $f \mapsto d$ is an ETI system with an infinite impulse response $g=g_{f} \oplus^{\prime} g_{b}$ :

$$
d=\left(f \oplus^{\prime} g_{f}\right) \oplus^{\prime} g_{b}=f \oplus^{\prime}\left(g_{f} \oplus^{\prime} g_{b}\right)=f \oplus^{\prime} g .
$$

The overall slope response,

$$
G(\mathbf{s})=G_{f}(\mathbf{s})+G_{b}(\mathbf{s})=G_{f}(\mathbf{s})+G_{f}(-\mathbf{s}),
$$


(a)

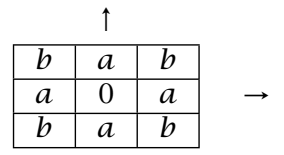

(c)

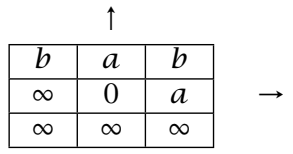

(b)

\begin{tabular}{|c|c|c|c|c|c|c|}
\multicolumn{1}{c|}{$\uparrow$} \\
\hline $3 b$ & $a+2 b$ & $2 a+b$ & $3 a$ & $2 a+b$ & $a+2 b$ & $3 b$ \\
\hline$a+2 b$ & $2 b$ & $a+b$ & $2 a$ & $a+b$ & $2 b$ & $a+2 b$ \\
\hline $2 a+b$ & $a+b$ & $b$ & $a$ & $b$ & $a+b$ & $2 a+b$ \\
\hline $3 a$ & $2 a$ & $a$ & 0 & $a$ & $2 a$ & $3 a$ \\
\hline $2 a+b$ & $a+b$ & $b$ & $a$ & $b$ & $a+b$ & $2 a+b$ \\
\hline$a+2 b$ & $2 b$ & $a+b$ & $2 a$ & $a+b$ & $2 b$ & $a+2 b$ \\
\hline $3 b$ & $a+2 b$ & $2 a+b$ & $3 a$ & $2 a+b$ & $a+2 b$ & $3 b$ \\
\hline
\end{tabular}

(d)

\begin{tabular}{c|c|c|c|c|c|c|}
\multicolumn{1}{c|}{$\uparrow$} \\
\hline $3 b$ & $a+2 b$ & $2 a+b$ & $3 a$ & $2 a+b$ & $a+2 b$ & $3 b$ \\
\hline$\infty$ & $2 b$ & $a+b$ & $2 a$ & $a+b$ & $2 b$ & $a+2 b$ \\
\hline$\infty$ & $\infty$ & $b$ & $a$ & $b$ & $a+b$ & $2 a+b$ \\
\hline$\infty$ & $\infty$ & $\infty$ & 0 & $a$ & $2 a$ & $3 a$ \\
\hline$\infty$ & $\infty$ & $\infty$ & $\infty$ & $\infty$ & $\infty$ & $\infty$ \\
\hline$\infty$ & $\infty$ & $\infty$ & $\infty$ & $\infty$ & $\infty$ & $\infty$ \\
\hline$\infty$ & $\infty$ & $\infty$ & $\infty$ & $\infty$ & $\infty$ & $\infty$ \\
\hline
\end{tabular}

Figure 10.10: Coefficient masks and impulse responses of ETI systems associated with computing the $(a, b)$ chamfer distance transform. (a) Local distances within the $3 \times 3$ pixel unit "disk." (b) Distances from origin by propagating three times the local distances in (a); also equal to a $7 \times 7$-pixel central portion of the infinite impulse response of the overall system associated with the distance transform. (c) Coefficient mask for the minsum difference equation computing the forward pass for the chamfer distance. (d) A $7 \times 7$ pixel portion of the infinite impulse response of the system corresponding to the min-sum difference equation computing the forward pass.

of this distance transform ETI system is the $0 /-\infty$ indicator function of a bounded convex region shown in Fig. 10.5c for $a=3, b=4$. Further, by using induction on $(i, j)$ and symmetry, we find that $g=g_{f} \oplus^{\prime} g_{b}$ is equal to the $(a, b)$ chamfer distance function

$$
g(i, j)=\|(i, j)\|_{a, b}
$$

A truncated version of $g$ is shown in Fig. 10.10b. Thus, our analysis has proved using ETI systems theory that the two-pass computation via recursive min-sum difference equations whose coefficients are the local chamfer distances yields the $(a, b)$ chamfer distance transform:

$$
\left[\mathrm{I}(S) \oplus^{\prime} \boldsymbol{g}_{f}\right] \oplus^{\prime} \mathfrak{g}_{b}=\mathrm{D}_{a, b}(S) .
$$

Two special cases are the well-known cityblock and chessboard discrete distances [Ros66]. The cityblock distance transform is obtained using $a=1$ and $b=+\infty$, that is, using the five-pixel rhombus as the unit "disk." It is an ETI system with impulse response $g(i, j)=|i|+|j|$ and the slope response being the indicator function of the unit square $\left\{\mathbf{s}:\|\mathbf{s}\|_{\infty}=1\right\}$. Similarly, the chessboard distance transform is obtained using $a=b=1$. It is an ETI system with impulse response $g(i, j)=\max (|i|,|j|)$ and the slope response being the indicator function of the unit rhombus $\left\{\mathbf{s}:\|\mathbf{s}\|_{1}=1\right\}$. 


\section{Parallel Computation and FIR Slope Filters}

The $(a, b)$ chamfer distance transform can be implemented alternatively using parallel operations. Namely, let

$$
g_{0}(i, j) \triangleq \begin{cases}g(i, j) & \text { for }|i|,|j| \leq 1 \\ +\infty & \text { otherwise }\end{cases}
$$

be the $3 \times 3$-pixel central portion of $g$ defined in Eq. (10.77). It can be shown via induction that the $n$ th-fold infimal convolution of $g_{0}$ with itself yields $g$ in the limit:

$$
g=\lim _{n \rightarrow \infty} \underbrace{\left(g_{0} \oplus^{\prime} g_{0}\right) \ldots \oplus^{\prime} g_{0}}_{n \text { times }} .
$$

Figure 10.10b shows the intermediate result for $n=3$ iterations. Similar finite decompositions of discrete conical functions into infimal convolutions of smaller kernels have been studied elsewhere [Shi91]. Consider now the nonautoregressive min-sum difference equation

$$
d_{n}(i, j)=\bigwedge_{k=-1}^{1} \bigwedge_{\ell=-1}^{1} g_{0}(i, j)+d_{n-1}(i-k, j-\ell),
$$

run iteratively for $n=1,2, \ldots$, starting from $d_{0}=f$. Each iteration is equivalent to the infimal convolution of the previous result with a finite impulse response equal to $g_{0}$. By iterating these local distances to the limit, the final distance transform is obtained: $d=\lim _{n \rightarrow \infty} d_{n}$. In practice, when the input image $f$ has finite support, the number of required iterations is finite and bounded by the image diameter.

\section{Optimal Chamfer Distance Transforms}

Selecting the steps $a, b$ under certain constraints leads to an infinite variety of chamfer metrics based on a $3 \times 3$ mask. The two well-known and easily computable special cases of the cityblock metric with $(a, b)=(1, \infty)$ and the chessboard metric with $(a, b)=(1,1)$ give the poorest discrete approximations to Euclidean distance (and to multiscale morphology with a disk structuring element), with errors reaching $41.4 \%$ and 29.3\%, respectively. Using Euclidean steps $(a, b)=$ $(1, \sqrt{2})$ yields a $7.61 \%$ maximum error. Thus, a major design goal is to reduce the approximation error between the chamfer distances and the corresponding Euclidean distances [Bor86]. A suitable error criterion is the maximum absolute error (MAE) between a unit chamfer ball and the corresponding unit disk [But98, Ver91]. The optimal steps obtained by Butt and Maragos [But98] for minimizing this MAE are $a=0.9619$ and $b=1.3604$, which give a $3.957 \%$ maximum error. In practice, for faster implementation, integer-valued distance steps $A$ and $B$ are used, and the computed distance transform is divided by a normalizing constant $k$, which can be real-valued. We refer to such a metric as $(a, b)=(A, B) / k$. Using two decimal digits for truncating optimal values and optimizing the triple $(A, B, k)$ 


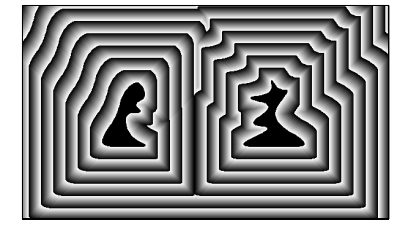

(a)

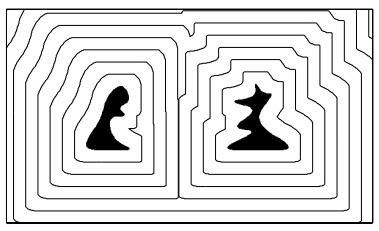

(d)

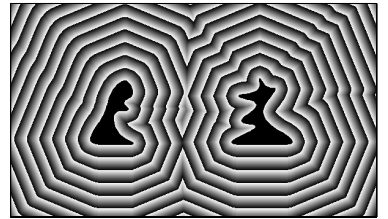

(b)

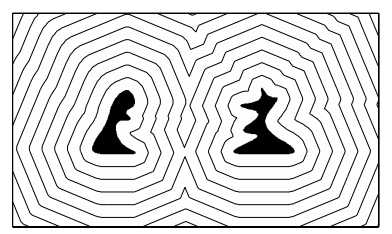

(e)

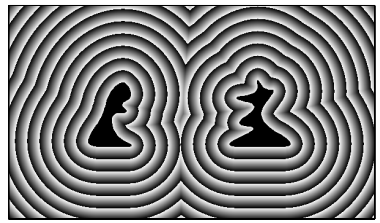

(c)

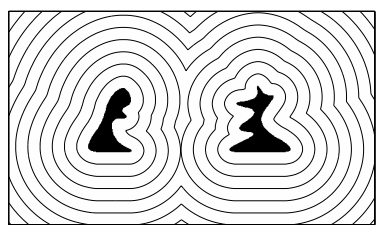

(f)

Figure 10.11: Top row: distance transforms of a binary image obtained via (a) a $(1,1)$ chamfer metric, (b) the optimal $3 \times 3$ chamfer metric, and (c) curve evolution. These distances are displayed as intensities modulo a constant $h=20$. Bottom row: the multiscale dilations (at scales $t=n h, n=1,2,3, \ldots$,) of the original set (filled black regions) were obtained by thresholding the three distance transforms at isolevel contours whose levels are multiples of $h$ using the following structuring elements: (d) and (e) the unit-scale polygons corresponding to the metrics used in (a) and (b) and (f) the disk. All images have a resolution $450 \times 600$ pixels.

as done by Butt and Maragos [But98] for the smallest possible error yields $A=70$, $B=99$, and $k=72.77$. The corresponding steps are

$$
(a, b)=(70,99) / 72.77
$$

yielding a 3.959\% MAE. See Fig. 10.11 for an example. By working as above, optimal steps that yield an even lower error can also be found for chamfer distances with a $5 \times 5$ mask or larger neighborhood [But98].

\subsection{Eikonal PDE and Distance Propagation}

The main postulate of geometrical optics [Bor59] is Fermat's principle of least time. Let us assume a 2D (i.e., planar) medium with (possibly space-varying) refractive index $\eta(x, y)=c_{0} / c(x, y)$, defined as the ratio of the speed $c_{0}$ of light in free space divided by its speed $c(x, y)$ in the medium. Given two points $A$ and $B$ in such a medium, the optical path length along a ray trajectory $\Gamma_{A B}$ (parameterized by $\ell$ ) between points $A$ and $B$ is

$$
\text { optical path length }=\int_{\Gamma_{A B}} \eta\left(\Gamma_{A B}(\ell)\right) \mathrm{d} \ell=c_{0} T\left(\Gamma_{A B}\right),
$$




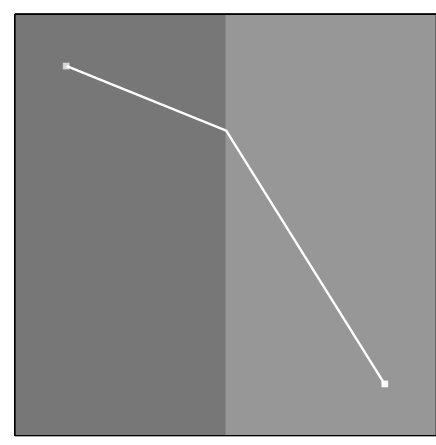

(a)

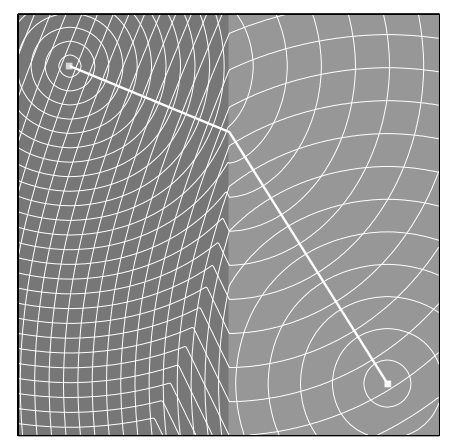

(b)

Figure 10.12: (a) Image of an optical medium consisting of two areas of different refractive indexes (whose ratio is 5/3) and the correct path of the light ray (from Snell's law) between two points. (b) Path found using the weighted distance function (numerically estimated via curve evolution); the thin light contours show the wavefronts propagating from the two source points.

where $\mathrm{d} \ell$ is the differential length element along this trajectory and $T\left(\Gamma_{A B}\right)$ is the time required for the light to travel this path. Fermat's principle states that light will choose a path between $A$ and $B$ that minimizes the optical path length.

An alternative viewpoint of geometrical optics is to consider the scalar function $E(x, y)$, called the eikonal, whose isolevel contours are normal to the rays. Thus, the eikonal's gradient $\|\nabla E\|$ is parallel to the rays. It can be shown [Bor59] using calculus of variations that Fermat's principle is equivalent to the following PDE:

$$
\|\nabla E(x, y)\|=\sqrt{\left(\frac{\partial E}{\partial x}\right)^{2}+\left(\frac{\partial E}{\partial y}\right)^{2}}=\eta(x, y),
$$

called the eikonal equation. Thus, the minimal optical path length between two points located at $A$ and $B$ is

$$
E(B)-E(A)=\inf _{\Gamma_{A B}} \int_{\Gamma_{A B}} \eta\left(\Gamma_{A B}(\ell)\right) \mathrm{d} \ell .
$$

Assume an optical wave propagating in a 2D medium of index $\eta(x, y)$ at wavelengths much smaller than the image objects, so that ray optics can approximate wave optics. Then, the eikonal $E$ of ray optics is proportional to the phase of the wavefunction. Hence, the isolevel contour lines of $E$ are the wavefronts. Assuming that at time $t=0$ there is an initial wavefront at a set of source points $S_{i}$, we can trace the wavefront propagation using Huygen's envelope construction: Namely, if we dilate the points $P=(x, y)$ of the wavefront curve at a certain time $t$ with circles of infinitesimal radius $c(x, y) \mathrm{d} t$, the envelope of these circles yields the wavefront at time $t+\mathrm{d} t$. If $T(P)$ is the time required for the wavefront to arrive at $P$ from the sources, then

$$
E(P)=c_{0} T(P)=\inf _{i}\left\{\inf _{\Gamma_{S_{i}} P} \int_{\Gamma_{S_{i} P}} \eta\left(\Gamma_{S_{i} P}(\ell)\right) \mathrm{d} \ell+E\left(S_{i}\right)\right\} .
$$


Thus, we can equate the eikonal $E(x, y)$ to the weighted distance function between a point $(x, y)$ and the sources along a path of minimal optical length and also view $E$ as proportional to the wavefront arrival time $T(x, y)$ (see also [Bor59, Kim96, Lev70, Rou92, Ver90]). An example is shown in Fig. 10.12.

Many tasks for extracting information from visible images have been related to optics and wave propagation via the eikonal PDE. Its solution $E(x, y)$ can provide shape from shading, analog contour-based halftoning, and topographic segmentation of an image by choosing the refractive index field $\eta(x, y)$ to be an appropriate function of the image brightness [Hor86, Kim96, Naj94, Pnu94, Sch83, Ver90]. Further, in the context of curve evolution, the eikonal PDE can be seen as a stationary formulation of the embedding level function evolution PDE of Eq. (10.60) with positive speed $V=\beta(x, y)=\beta_{0} / \eta(x, y)>0$. Namely, as explained elsewhere [Bar90, Fal94, Osh88]), if

$$
T(x, y)=\inf \{t: \phi(x, y, t)=0\}
$$

is the minimum time at which the zero-level curve of $\phi(x, y, t)$ crosses $(x, y)$, then

$$
\|\nabla T(x, y)\|=\frac{1}{\beta(x, y)} .
$$

Setting $E=\beta_{0} T$ leads to the eikonal.

In short, we can view the solution $E(x, y)$ of the eikonal as a weighted distance transform (WDT) whose values at each pixel give the minimum distance from the light sources weighted by the gray values of the refractive index field. On a computational grid this solution is numerically approximated using discrete WDTs, which can be implemented either via 2D recursive min-sum difference equations or via numerical algorithms of curve evolution. The former implementation employs adaptive 2D recursive erosions and is a stationary approach to solving the eikonal, whereas the latter involves a time-dependent formulation and evolves curves based on a dilation-type PDE at a speed varying according to the gray values. Next we outline these two ways of solving the eikonal PDE and discuss some of its applications.

\section{WDT Based on Chamfer Metrics}

Let $f(i, j) \geq 1$ be a sampled nonnegative graylevel image and let us view it as a discrete refractive index field. Also let $S$ be a set of reference points or the "sources" of some wave or the location of the wavefront at time $t=0$. The discrete WDT finds at each pixel $P=(i, j)$ the smallest sum of values of $f$ over all possible discrete paths connecting $P$ to the sources $S$. It can also be viewed as a procedure for finding paths of minimal "cost" among nodes of a weighted graph or as discrete dynamic programming. It has been used extensively in image analysis problems such as minimal path finding, weighted distance propagation, and graylevel image skeletonization; for example, see [Lev70, Mey92, Rut68, Ver91].

The above discrete WDT can be computed by running a 2D min-sum difference equation like Eq. (10.72) that implements the chamfer distance transform of binary 
images but with spatially varying coefficients proportional to the gray image values [Rut68, Ver90]:

$$
\begin{aligned}
u_{n}(i, j)= & \min \left\{u_{n}(i-1, j)+a f(i, j),\right. \\
& u_{n}(i, j-1)+a f(i, j), u_{n}(i-1, j-1)+b f(i, j), \\
& \left.u_{n}(i+1, j-1)+b f(i, j), u_{n-1}(i, j)\right\},
\end{aligned}
$$

where $u_{0}=\mathrm{I}(S)$ is the $0 / \infty$ indicator function of the source set $S$. Starting from $u_{0}$, a sequence of functions $u_{n}$ is iteratively computed by running Eq. (10.88) over the image domain in a forward scan for even $n$, whereas for odd $n$ an equation similar to Eq. (10.88) but with a reflected coefficient mask is run in a backward scan. In the limit $n \rightarrow \infty$ the final WDT $u_{\infty}$ is obtained. In practice, this limit is reached after a finite number of passes. The number of iterations required for convergence depends on both the sources and the gray values. There are also other, faster implementations using queues (see [Mey92, Ver90]). The final transform is a function of the source set $S$, the index field, and the norm used for horizontal distances.

The above WDT based on discrete chamfer metrics is a discrete approximate solution of the eikonal PDE. The rationale for such a solution is that, away from the sources, this difference equation mapping $f \mapsto u$ corresponds to

$$
\bigvee_{(k, \ell) \in B} \frac{u(i, j)-u(i-k, j-\ell)}{a_{i j}}=f(i, j)
$$

where $B$ is equal to the union of the output mask and its reflection and $a_{i j}$ are the chamfer steps inside $B$. The left side of Eq. (10.89) is a weighted discrete approximation to the morphological derivative $\mathcal{M}(-u)$ with horizontal distances weighted by $a_{i j}$. Thus, since in the continuous case $\mathcal{M}(-u)=\|\nabla u\|$, Eq. (10.89) is an approximation of the eikonal. In fact, as established elsewhere [Mey92], it is possible to recover a digital image $u$ from its half morphological gradient $u-u \ominus B$ using the discrete weighted distance transform if one uses 1-pixel sources in each regional minimum of $u$.

The constants $a$ and $b$ in Eq. (10.88) are the distance steps by which the planar chamfer distances are propagated within a $3 \times 3$ neighborhood. The propagation of the local distances $(a, b)$ starts at the points of sources $S$ and moves with speed $V=\beta(i, j)=\beta_{0} / f(i, j)$. If $f$ is a binary image, then the propagation speed is constant and the solution of the above difference equation (after convergence) yields the discrete chamfer distance transform of $S$. To improve the WDT approximation to the eikonal's solution, one can optimize $(a, b)$ so that the error is minimized between the planar chamfer distances and the Euclidean distances. Using a neighborhood larger than $5 \times 5$ can further reduce the approximation error but at the cost of an even slower implementation. However, larger neighborhood masks cannot be used with WDTs because they give erroneous results since the large masks can bridge over a thin line that separates two segmentation regions. Overall, this chamfer metric approach to WDT is fast and easy to implement but due to the required small neighborhoods is not isotropic and cannot achieve high accuracy. 


\section{WDT Based on Curve Evolution}

In this approach, at time $t=0$ the boundary of each source is modeled as a curve $\gamma(0)$ which is then propagated with normal speed $V=\beta(x, y)=\beta_{0} / \eta(x, y)$. The propagating curve $\gamma(t)$ is embedded as the zero-level curve of a function $\phi(x, y, t)$, where $\phi(x, y, 0)=\phi_{0}(x, y)$ is the signed (positive in the curve interior) distance from $\gamma(0)$. The function $\phi$ evolves according to the PDE

$$
\phi_{t}=\beta(x, y)\|\nabla \phi\|,
$$

which corresponds to curve evolution in a heterogeneous medium with positiondependent speed $\beta>0$, or equivalently to a successive front dilation by disks with position-varying radii $\beta(x, y) \mathrm{d} t$. This is a time-dependent formulation of the eikonal PDE [Fal94, Osh88]. It can be solved via Osher and Sethian's numerical algorithm given by Eq. (10.49). The value of the resulting WDT at any pixel $(x, y)$ of the image is the time it takes for the evolving curve to reach this pixel, that is, the smallest $t$ such that $\phi(x, y, t) \geq 0$. This continuous approach to the WDT can achieve subpixel accuracy, as investigated by Kimmel et al. [Kim96]. In the applications of the eikonal PDE examined herein, the global speed function $\beta(x, y)$ is everywhere nonnegative. In such cases the computational complexity of Osher and Sethian's level set algorithm (which can handle sign changes in the speed function) can be significantly reduced by using Sethian's fast marching algorithm [Set96], which is designed to solve the corresponding stationary formulation of the eikonal PDE, that is, $\|\nabla T\|=1 / \beta$. There are also other types of numerical algorithms for solving stationary eikonal PDEs; for example, Rouy and Tourin [Rou92] have proposed an efficient iterative algorithm for solving $\|\nabla E\|=\eta$.

All of the numerical image experiments with curve evolution shown herein were produced using an implementation of fast marching based on a simple data structure of two queues. This data structure is explained in [Mar00] and has been used to implement WDTs based on either chamfer metrics or fast marching for applications both with single sources as well as with multiple sources, where triple points develop at the collision of several wavefronts.

\section{Gridless Halftoning via the Eikonal PDE}

Inspired by the use in Schröder [Sch83] of the eikonal function's contour lines for visually perceiving an intensity image $I(x, y)$, Verbeek and Verwer [Ver90] and especially Pnueli and Bruckstein [Pnu94] attempted to solve the PDE

$$
\|\nabla E(x, y)\|=\text { constant }-I(x, y)
$$

and create a binary gridless halftone version of $I(x, y)$ as the union of the level curves of the eikonal function $E(x, y)$. The larger the intensity value $I(x, y)$, the smaller the local density of these contour lines in the vicinity of $(x, y)$. This eikonal PDE approach to gridless halftoning, which we call eikonal halftoning, is indeed very promising and can simulate various artistic effects, as shown in 


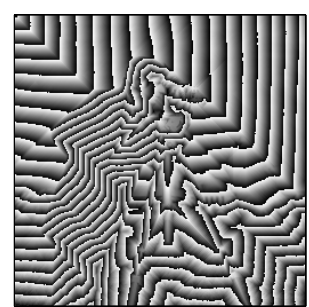

(a)

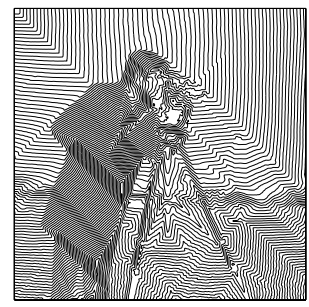

(d)

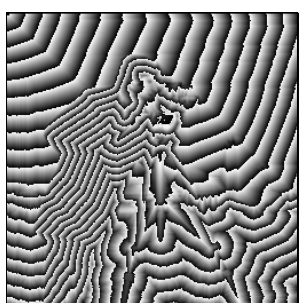

(b)

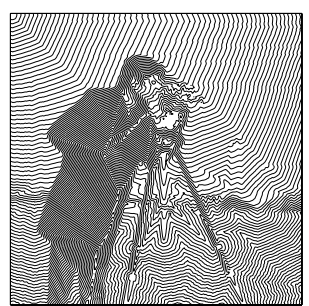

(e)

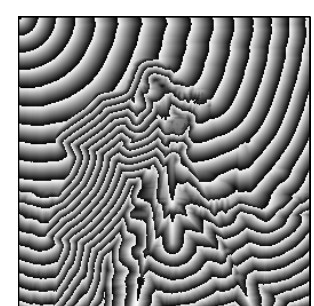

(c)

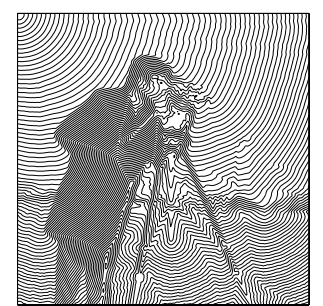

(f)

Figure 10.13: Eikonal halftoning of the Cameraman image $I$ from the weighted distance transform of the "negative" image $\max (I)-I$. Top row: the light source was at the top left corner, and the WDTs (displayed as intensities modulo a height such that 25 waves exist per image) were obtained via (a) a $(1,1)$ chamfer metric, (b) the optimal $3 \times 3$ chamfer metric, and (c) curve evolution. Bottom row: 100 contour lines of the WDTs in the top row give gridless halftoning of the original images.

Fig. 10.13, which also shows that the curve evolution WDT gives a smoother halftoning of the image than the WDTs based on chamfer metrics.

\section{Watershed Segmentation via the Eikonal}

A powerful morphological approach to image segmentation is the watershed algorithm [Mey90, Vin91b], which transforms an image $f(x, y)$ to the crest lines separating adjacent catchment basins that surround regional minima or other "marker" sets of feature points. Najman and Schmitt [Naj94] established that (in the continuous domain and assuming that the image is smooth and has isolated critical points) the continuous watershed is equivalent to finding a skeleton by influence zones with respect to a weighted distance function that uses points in the regional minima of the image as sources and $\|\nabla f\|$ as the field of indices. A similar result was obtained by Meyer [Mey94] for digital images. In Maragos and Butt [Mar00] the eikonal PDE modeling the watershed segmentation of an image-related function $f$ was solved by finding a WDT via the curve evolution PDE of Eq. (10.90) in which the speed $\beta$ is proportional to $1 /\|\nabla f\|$. Further, the results of this new segmentation approach [Mar00] have been compared to the digital watershed algorithm via flooding [Vin91b] and to the eikonal approach solved via a discrete WDT based on chamfer metrics [Mey94, Ver90]. In all three approaches, robust features are extracted first as markers of the regions, and the original image $I$ is trans- 


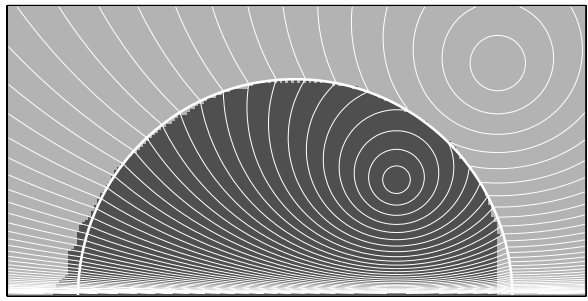

(a)

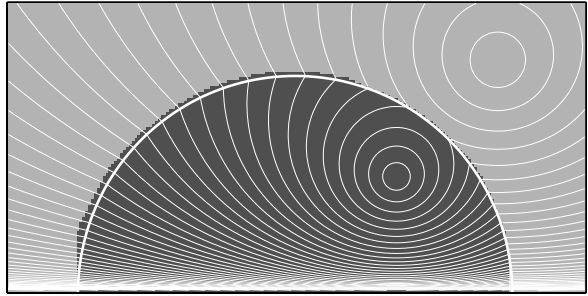

(c)

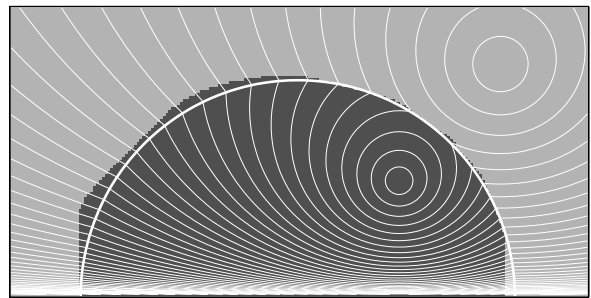

(b)

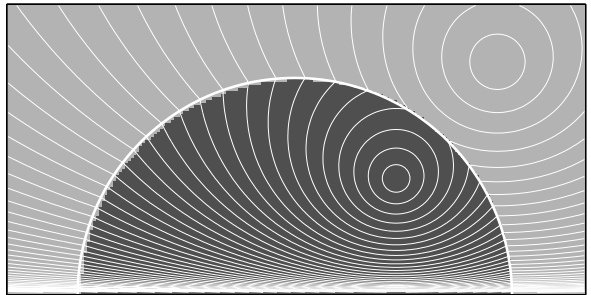

(d)

Figure 10.14: Performance of various segmentation algorithms on a Test image, $250 \times 400$ pixels that is the minimum of two potential functions. Its contour plot (thin bright curves) is superimposed on all segmentation results. Markers are the two source points of the potential functions. The segmentation results are from (a) the digital watershed flooding algorithm and from WDTs based on (b) the optimal $3 \times 3$ chamfer metric, (c) the optimal $5 \times 5$ chamfer metric, and (d) curve evolution. (The thick bright curve shows the correct segmentation.)

formed to another function $f$ by smoothing via alternating opening-closing by reconstruction, taking the gradient magnitude of the filtered image, and changing (via morphological reconstruction) the homotropy of the gradient image so that its only minima occur at the markers. The segmentation is done on the final outcome $f$ of the above processing.

In the standard digital watershed algorithm via flooding [Mey90, Vin91b], the flooding at each level is achieved by a planar distance propagation that uses the chessboard metric. This kind of distance propagation is not isotropic and could give wrong results, particularly for images with large plateaus. Eikonal segmentation using WDTs based on chamfer metrics improves this situation a little but not entirely. In contrast, for images with large plateaus or regions, segmentation via the eikonal PDE and curve evolution WDT gives results close to ideal. Using a test image that was difficult (because expanding wavefronts meet watershed lines at many angles ranging from being perpendicular to almost parallel), Fig. 10.14 shows that the continuous segmentation approach based on the eikonal PDE and curve evolution outperforms the discrete segmentation results (using either the digital watershed flooding algorithm or chamfer metric WDTs). In real images, which may contain a large variety of region sizes and shapes, the digital watershed flooding algorithm may give results comparable to the eikonal PDE approach. Details on comparing the two segmentation approaches can be found in [Mar00]. 


\subsection{Conclusions}

We have provided a unified view and some analytic tools for a recently growing part of morphological image processing that is based on ideas from differential calculus and dynamic systems, including the use of partial differential equations or difference equations to model nonlinear multiscale analysis or distance propagation in images. We have discussed general 2D nonlinear difference equations of the max-sum or min-sum type that model the space dynamics of 2D morphological systems (including the distance computations) and some nonlinear signal transforms, called slope transforms, that can analyze these systems in a transform domain in ways conceptually similar to the application of Fourier transforms to linear systems. We have used these nonlinear difference equations to model discrete distance transforms and relate them to numerical solutions of the eikonal PDE of optics. In this context, distance transforms are shown to be bandpass slope filters. We have also reviewed some nonlinear PDEs that model the evolution of multiscale morphological operators and use morphological derivatives. Related to these morphological PDEs is the area of curve evolution, which employs methods of differential geometry to study the differential equations governing the propagation of time-evolving curves. The PDEs governing multiscale morphology and most cases of curve evolution are of the Hamilton-Jacobi type and are related to the eikonal PDE of optics.

We view the analysis of the multiscale morphological PDEs and of the eikonal PDE solved via weighted distance tranforms as a unified area in nonlinear image processing that we call differential morphology, and we have briefly discussed some of its potential applications to image processing. 


\section{References}

[Alv92] L. Alvarez, F. Guichard, P.L. Lions, and J.M. Morel. Axiomatization et nouveaux operateurs de la morphologie mathematique. C. R. Acad. Sci. Paris 315, Series I, 265-268 (1992).

[Alv93] L. Alvarez, F. Guichard, P.L. Lions, and J.M. Morel. Axioms and fundamental equations of image processing. Archiv. Rat. Mech. 123(3), 199-257 (1993).

[Are93] A. Arehart, L. Vincent and B. Kimia. Mathematical morphology: the Hamilton-Jacobi connection. In Proc. Intl. Conf. on Computer Vision, pp. 215219 (1993).

[Bar90] M. Bardi and M. Falcone. An approximation scheme for the minimum time function. SIAM J. Control Optimization 28, 950-965 (1990).

[Blu73] H. Blum. Biological shape and visual science (part I). J. Theoret. Biol. 38, 205-287 (1973).

[Boo92] R. van den Boomgaard. Mathematical morphology: extensions towards computer vision. Ph.D. thesis, Univ. of Amsterdam, The Netherlands (1992).

[Boo94] R. van den Boomgaard and A. Smeulders. The morphological structure of images: the differential equations of morphological scale-space. IEEE Trans. Pattern Anal. Mach. Intellig. 16, 1101-1113 (November 1994).

[Bor59] M. Born and E. Wolf. Principles of Optics. Pergamon Press, Oxford, England (1959; 1987 edition).

[Bor86] G. Borgefors. Distance transformations in digital images. Comput. Vision Graph. Image Process. 34, 344-371 (1986).

[Bro92] R. W. Brockett and P. Maragos. Evolution equations for continuous-scale morphology. In Proc. IEEE Intl. Conf. on Acoustics, Speech, and Signal Processing (San Francisco, CA, March 1992).

[Bro94] R. Brockett and P. Maragos. Evolution equations for continuous-scale morphological filtering. IEEE Trans. Signal Process. 42, 3377-3386 (December 1994).

[But98] M. A. Butt and P. Maragos. Optimal design of chamfer distance transforms. IEEE Trans. Image Process. 7, 1477-1484 (October 1998).

[Che89] M. Chen and P. Yan. A multiscaling approach based on morphological filtering. IEEE Trans. Pattern Anal. Machine Intell. 11, 694-700 (July 1989).

[Cou62] R. Courant and D. Hilbert. Methods of Mathematical Physics, Wiley, New York (1962). 
[Cra92] M. G. Crandall, H. Ishii, and P.-L. Lions. User's guide to viscosity solutions of second order partial differential equations. Bull. Amer. Math. Soc. 27,1-66 (July 1992).

[Dan80] P.-E. Danielsson, Euclidean distance mapping. Comp. Graph. Image Process. 14, 227-248 (1980).

[Dor94] L. Dorst and R. van den Boomgaard. Morphological signal processing and the slope transform. Signal Process. 38, 79-98 (July 1994).

[Dud84] D. E. Dudgeon and R. M. Mersereau. Multidimensional Digital Signal Processing. Prentice Hall, New Jersey (1984).

[Fal94] M. Falcone, T. Giorgi, and P. Loreti. Level sets of viscocity solutions: some applications to fronts and rendez-vous problems. SIAM J. Appl. Math. 54, 1335-1354 (October 1994).

[Hei94] H. J. A. M. Heijmans. Morphological Image Operators, Academic Press, Boston (1994).

[Hei97] H. J. A. M. Heijmans and P. Maragos. Lattice calculus and the morphological slope transform. Signal Process. 59, 17-42 (1997).

[Hor86] B. K. P. Horn. Robot Vision. MIT Press, Cambridge, MA (1986).

[Hua94] C. T. Huang and O. R. Mitchell. A euclidean distance transform using grayscale morphology decomposition. IEEE Trans. Pattern Anal. Machine Intell. 16, 443-448 (April 1994).

[Kim90] B. Kimia, A. Tannenbaum, and S. Zucker. Toward a computational theory of shape: an overview. In Proc. European Conf. on Computer Vision (France, April 1990).

[Kim96] R. Kimmel, N. Kiryati, and A. M. Bruckstein. Sub-pixel distance maps and weighted distance transforms. J. Mathemat. Imaging Vision 6, 223-233 (1996).

[Koe84] J. J. Koenderink. The structure of images. Biolog. Cybern. 50, 363-370 (1984).

[Lax73] P. D. Lax. Hyperbolic Systems of Conservation Laws and the Mathematical Theory of Schock Waves. SIAM, Philadelphia (1973).

[Lev70] G. Levi and U. Montanari. A grey-weighted skeleton. Inform. Control 17, 62-91 (1970).

[Mar89] P. Maragos. Pattern spectrum and multiscale shape representation. IEEE Trans. Pattern Anal. Machine Intell. 11, 701-716 (July 1989).

[Mar94] P. Maragos. Morphological systems: slope transforms and max-min difference and differential equations. Signal Process. 38, 57-77 (July 1994). 
[Mar96] P. Maragos. Differential morphology and image processing. IEEE Trans. Image Process. 78, 922-937 (June 1996).

[Mar98] P. Maragos. Morphological signal and image processing. In The Digital Signal Processing Handbook (V. Madisetti and D. Williams, eds.) CRC and IEEE Press (1998).

[Mar00] P. Maragos and M. A. Butt. Curve evolution, differential morphology, and distance transforms applied to multiscale and eikonal problems. Fundamenta Informaticae 41, 91-129 (2000).

[Mar99] P. Maragos and F. Meyer. Nonlinear PDEs and numerical algorithms for modeling levelings and reconstruction filters. In Scale-Space Theories in Computer Vision (Proc. Intl. Conf. Scale-Space'99) pp. 363-374. Lecture Notes in Computer Science Vol. 1682. Springer-Verlag (1999).

[Mar90] P. Maragos and R. W. Schafer. Morphological systems for multidimensional signal processing. Proc. IEEE 78, 690-710 (April 1990).

[Mar82] D. Marr. Vision. Freeman, San Francisco (1982).

[Mat75] G. Matheron. Random Sets and Integral Geometry. Wiley, New York (1975).

[Mat93] J. Mattioli. Differential relations of morphological operators. In Proc. Intl. Workshop on Mathematical Morphology and Its Application to Signal Processing (J. Serra and P. Salembier, eds.). Univ. Polit. Catalunya, Barcelona, Spain (1993).

[Mey92] F. Meyer. Integrals and gradients of images. In Image Algebra and Morphological Image Processing III. Proc. SPIE Vol. 1769, pp. 200-211 (1992).

[Mey94] F. Meyer. Topographic distance and watershed lines. Signal Process. 38, 113-125 (July 1994).

[Mey98] F. Meyer. The levelings. In Mathematical Morphology and Its Applications to Image and Signal Processing (H. Heijmans and J. Roerdink, eds.), pp. 199-206, Kluwer Acad. Publ. (1998).

[Mey90] F. Meyer and S. Beucher. Morphological segmentation. J. Visual Commun. Image Representation 1(1), 21-45 (1990).

[Nac96] P. F. M. Nacken. Chamfer metrics, the medial axis and mathematical morphology. J. Mathemat. Imaging Vision 6, 235-248 (1996).

[Naj94] L. Najman and M. Schmitt. Watershed of a continuous function. Signal Process. 38, 99-112 (July 1994).

[Osh88] S. Osher and J. Sethian. Fronts propagating with curvature-dependent ppeed: algorithms based on Hamilton-Jacobi formulations. J. Comput. Phys. 79, 12-49 (1988). 
[Pnu94] Y. Pnueli and A. M. Bruckstein. Digi ${ }_{D}$ ürer-a digital engraving system. Visual Comput. 10 277-292 (1994).

[Pre93] F. Preteux. On a distance function approach for gray-level mathematical morphology. In Mathematical Morphology in Image Processing (E.R. Dougherty, ed.) Marcel Dekker, New York (1993).

[Roc70] R. T. Rockafellar. Convex Analysis. Princeton Univ. Press (1970).

[Ros66] A. Rosenfeld and J. L. Pfaltz. Sequential operations in digital picture processing. J. ACM 13, 471-494 (October 1966).

[Ros68] A. Rosenfeld and J. L. Pfaltz. Distance functions on digital pictures. Pattern Recog. 1, 33-61 (1968).

[Rou92] E. Rouy and A. Tourin. A viscocity solutions approach to shape from shading. SIAM J. Numer. Anal. 29(3), 867-884 (June 1992).

[Rut68] D. Rutovitz. Data structures for operations on digital images. In Pictorial Pattern Recognition (G.C. Cheng et al. eds.), pp. 105-133. Thompson, Washington, DC (1968).

[Sal95] P. Salembier and J. Serra. Flat zones filtering, conencted operators, and filters by reconstruction. IEEE Trans. Image Process. 4, 1153-1160 (August 1995).

[Sap93] G. Sapiro, R. Kimmel, D. Shaked, B. Kimia, and A. Bruckstein. Implementing continuous-scale morphology via curve evolution. Pattern Recog. 26(9), 1363-1372 (1993).

[Sch83] M. Schröder. The eikonal equation. Math. Intelligencer 1, 36-37 (1983).

[Ser82] J. Serra. Image Analysis and Mathematical Morphology, Academic Press, New York (1982).

[Ser88] J. Serra (ed.). Image Analysis and Mathematical Morphology, Vol. 2. Academic Press, New York (1988).

[Set96] J. A. Sethian. Level Set Methods. Cambridge Univ. Press (1996).

[Shi91] F. Y.-C. Shih and O. R. Mitchell. Decomposition of gray-scale morphological structuring elements. Pattern Recog. 24, 195-203 (1991).

[Ste80] S. R. Sternberg. Language and Architecture for Parallel Image Processing. In Pattern Recognition in Practice (E. S. Gelsema and L. N. Kanal, eds.). North Holland Publ. (1980).

[Tek98] H. Tek and B. B. Kimia. Curve evolution, wave propagation, and mathematical morphology. In Mathematical Morphology and Its Applications to Image and Signal Processing (H. Heijmans and J. Roerdink, eds.), pp. 115-126, Kluwer (1998). 
[Ver90] P. Verbeek and B. Verwer. Shading from shape, the eikonal equation solved by grey-weighted distance transform. Pattern Recog. Lett. 11, 618-690 (1990).

[Ver91] B. J. H. Verwer. Distance transforms: metrics, algorithms, and applications. Ph.D. thesis, Tech. Univ. of Delft, The Netherlands (1991).

[Vin91a] L. Vincent. Exact Euclidean distance function by chain propagations. In Proc. Conf. on Computer Vision and Pattern Recognition, pp. 520-525 (1991).

[Vin91b] L. Vincent and P. Soille. Watershed in digital spaces: an efficient algorithm based on immersion simulations. IEEE Trans. Pattern Anal. Machine Intell. 13, 583-598 (June 1991).

[Vin93] L. Vincent. Morphological grayscale reconstruction in image analysis: applications and efficient algorithms. IEEE Trans. Image Process. 2, 176-201 (April 1993).

[Wit83] A. P. Witkin. Scale-space filtering. Proc. Intl. Joint Conf. on Artificial Intelligence (Karlsruhe, Germany, 1983). 